\title{
SURFACE ACOUSTIC WAVE SENSING OF VOCS IN HARSH CHEMICAL ENVIRONMENTS
}

\author{
by \\ K. B. Pfeifer, S. J. Martin, and A. J. Ricco \\ Microsensor Research Department, 1315 \\ Sandia National Laboratories \\ Albuquerque, NM 87185
}

\begin{abstract}
The measurement of VOC concentrations in harsh chemical and physical environments is a formidable task. A surface acoustic wave (SAW) sensor has been designed for this purpose and its construction and testing are described in this paper. Included is a detailed description of the design elements specific to operation in $300^{\circ} \mathrm{C}$ steam and $\mathrm{HCl}$ environments including temperature control, gas handling, and signal processing component descriptions. In addition, laboratory temperature stability was studied and a minimum detection limit was defined for operation in industrial environments. Finally, a description of field tests performed on steam reforming equipment at Synthetica Technologies Inc. of Richmond, CA is given including a report on destruction efficiency of $\mathrm{CCl}_{4}$ in the Synthetica moving bed evaporator. Design improvements based on the field tests are proposed.
\end{abstract}




\section{TABLE OF CONTENTS}

PAGE

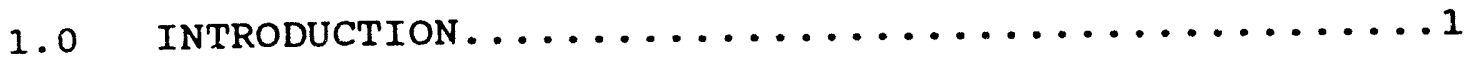

2.0 SURFACE ACOUSTIC WAVE SENSORS.............. 5

2.1 PERTURBATIONS IN ACOUSTIC WAVE PROPAGATION.......7

2.2 SURFACE ACOUSTIC WAVE (SAW) DEVICES........... 1

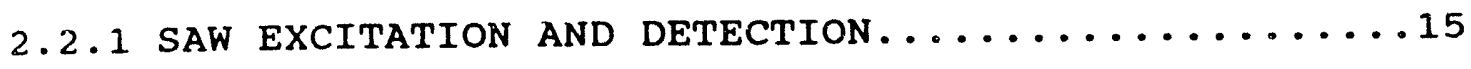

2.2.2 INTERDIGITAL TRANSDUCER FREQUENCY RESPONSE......16

2.2 .3 SAW PERTURBATION MECHANISMS............... 20

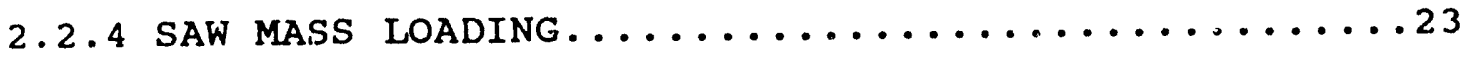

2.2 .5 SUMMARY OF SAW PROPERTIES.....................

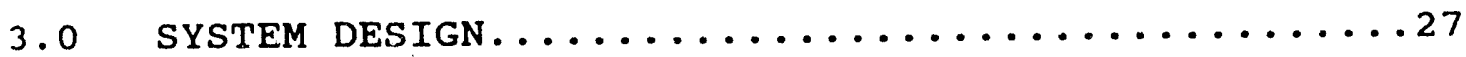

3.1 SAW OSCILLATOR CIRCUIT.......................

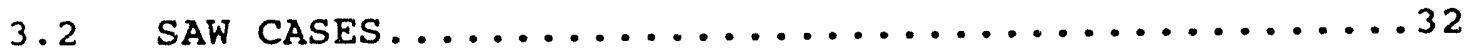

3.3 MIXER SIGNAL PROCESSOR CIRCUIT............... 35

3.4 HEATER CIRCUITS........................ 37

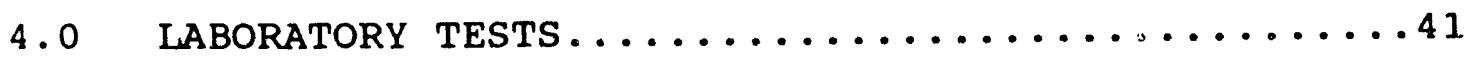

4.1 POLYISOBUTYLENE FILMS................4

4.2 TEMPERATURE STABILITY............... 42

$5.0 \quad$ FIELD TESTS ........................ 48

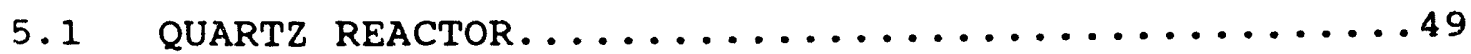

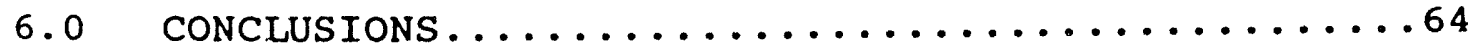

APPENDIX A........................68

REFERENCES......................... 


\section{LIST OF FIGURES}

FIGURE

PAGE

1.1. Pictorial diagram of surface acoustic wave (SAW) VOC sensor..........4

2.1. Cross section of a quartz substrate......14

2.2. Relationship between IDT periodicity and coherently excited waves...............

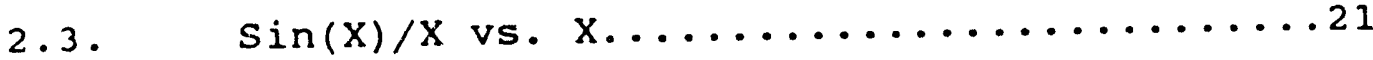

2.4. Relative insertion loss as a function of frequency.............22

2.5. Surface acoustic wave device normalized velocity shift and attenuation shift

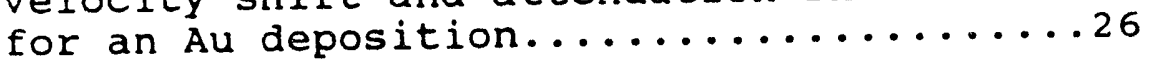

3.1 . Block diagram of SAW VOC sensor system...29

3.2 . Schematic diagram of SAW Oscillator

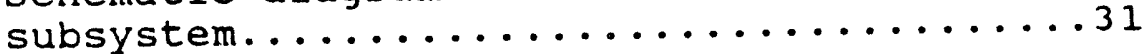

3.3 . Pictorial diagram of SAW case............

3.4 . Schematic diagram of signal processing

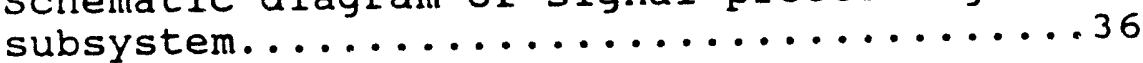

3.5 . Schematic diagram of temperature control

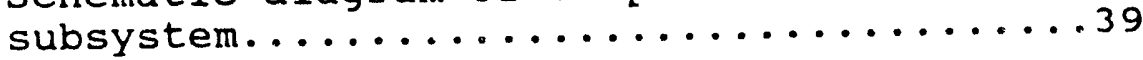

4.1. Graph showing long-term stability of SAW

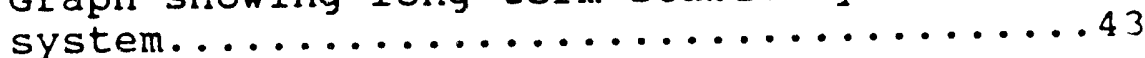

4.2. Pictorial diagram of $\mathrm{CCl}_{4}$ calibration flow system.....................46

4.3. $\mathrm{CCl}_{4}$ in $\mathrm{N}_{2}$ concentration as a function of frequency change...............47

5.1. Pictorial diagram of synthetica quartz reactor and flow system................. 50

5.2 .

Frequency response of SAW sensor as a function of time in quartz reactor.......52 


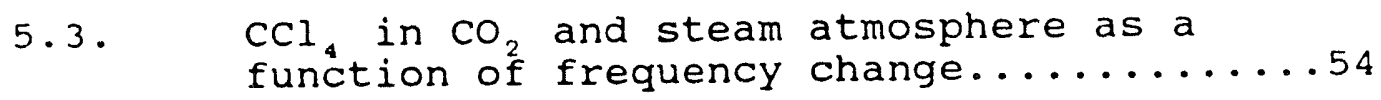

5.4. Change in SAW frequency and corresponding change in $\mathrm{CCl}_{4}$ concentration as a function of quartz reactor temperature..........55

5.5. Schematic diagram of synthetica MBEV, STD, and Sandia SAW sensor...................

5.6. Measured $\mathrm{CCl}_{4}$ outlet concentration normalized to calculated $\mathrm{CCl}_{4}$ inlet concentration as a function of MBEv outlet temperature..62

A.1. Mechanical drawing of reference

SAW test case...................68

A.2. Mechanical drawing of signal

SAw test case.....................69

A.3. Mechanical drawing of test case lid.....70

A.4. Mechanical drawing of Teflon bushing.....71 


\section{SURFACE ACOUSTIC WAVE SENSING OF VOCS IN HARSH CHEMICAL ENVIRONMENTS}

\subsection{INTRODUCTION}

Much attention has been paid recently to the collateral damage that has been done to the environment as a result of the cold war. In addition to the well known environmental disaster sites at Hanford (WA), Savannah River (SC), and Rocky Flats (CO), there are literally hundreds of other sites around the United States where soil and ground-water contamination has occurred. In fact, any site where high-technology materials and devices are produced has the potential for soil and ground-water contamination due to the volume of volatile organic compounds (VOCs) associated with those industries.

It has become clear that in order to restore the environment and protect the public health, it will be necessary to cease ihe practices that have resulted in this contamination. In addition to the development of environmentally conscious manufacturing techniques, the old damage must be repaired. A whole range of environmental sensors must be developed to help assess the level and extent of the damage and evaluate the remediation technologies. This report is a description of a sensor developed for such a purpose.

One current method of cleaning up VOC contamination of ground water is called in-situ air stripping and involves the orilling of wells into the water table that are used to pump air down to 
the contamination, and then to pull the air and the volatilized contaminant out of the ground. The exhaust stream is then scrubbed of the contaminant by passing it through beds of granular activated charcoal (GAC) before it is released into the atmosphere. Eventually, the charcoal becomes saturated with the contaminant and the efficiency of the process diminishes. At this point, the charcoal is replaced and the process continued.

Replacement of the charcoal is undesirable because of the cost of new GAC $(\$ 2 / 1 b)$ and because the old GAC (loaded with the contaminant) is classified as hazardous waste and must be disposed of as such. In the past, reactivation of the charcoal has not been a viable option due to the inefficiency and high cost of the processes; however, a relatively efficient, low cost process has been developed by synthetica Technologies, Inc. to remove the organic waste from the charcoal and reactivate the charcoal absorber. This process is called the synthetica Technologies Detoxifier (STD) and uses steam to oxidize the organic chemicals to $\mathrm{CO}_{2}$ and $\mathrm{H}_{2} \mathrm{O}$, which can then be vented into the atmosphere. 1 The process works effectively on organics without heteroatoms (i.e. organics with only carbon, hydrogen, and oxygen); organics with heteroatoms, such as halogenated solvents (e.g. contain chlorine or fluorine), are reformed into $\mathrm{CO}_{2}, \mathrm{H}_{2} \mathrm{O}$ and mineral acids such as $\mathrm{HCl}$ or $\mathrm{HF}$. The acids eventually destroy the stainless steel vessel and heating elements used in the construction of the STD. This problem has been addressed by the development of a moving-bed evaporator 
(MBEV), that neutralizes the acid from the waste stream by passing it through a bed of spheres coated with a molten base such as $\mathrm{NaOH}$.

In order to evaluate the efficiency of the MBEv at destroying Vocs, it was necessary to develop a sensor to measure the concentration of $\mathrm{VOC}$ vapors in the exhaust stream of the MBEV. The exhaust stream from the MBEv is a high-temperature, potentially corrosive, steam environment that requires special engineering effort to measure the concentration of vocs without damaging the sensor.

The sensor is based on surface acoustic wave (SAW) technology.2 A polyisobutylene (PIB) film is spin cast onto the SAW device $(2000 \AA)$ to absorb species from the gas phase. The SAW device is used as the feedback element in an oscillator circuit whose frequency is then monitored as a function of time (F. jure 1.1). The amount of vocs absorbed by the polyisobutylene film is proportional to their gas-phase concentration; absorbed Vocs change the film's mass density and viscoelastic properties, resulting in changes in the acoustic wave velocity and hence the oscillator frequency. 3

A prototype sensor unit was assembled that included the SAW sensor, oscillator electronics, on-board SAW test case, and device temperature controller. The SAW case was designed to allow diffusion of the gas from the waste stream to the SAW sensor; sufficient distance was allowed for the hot waste stream sample to cool sufficiently before reaching the sensor, 


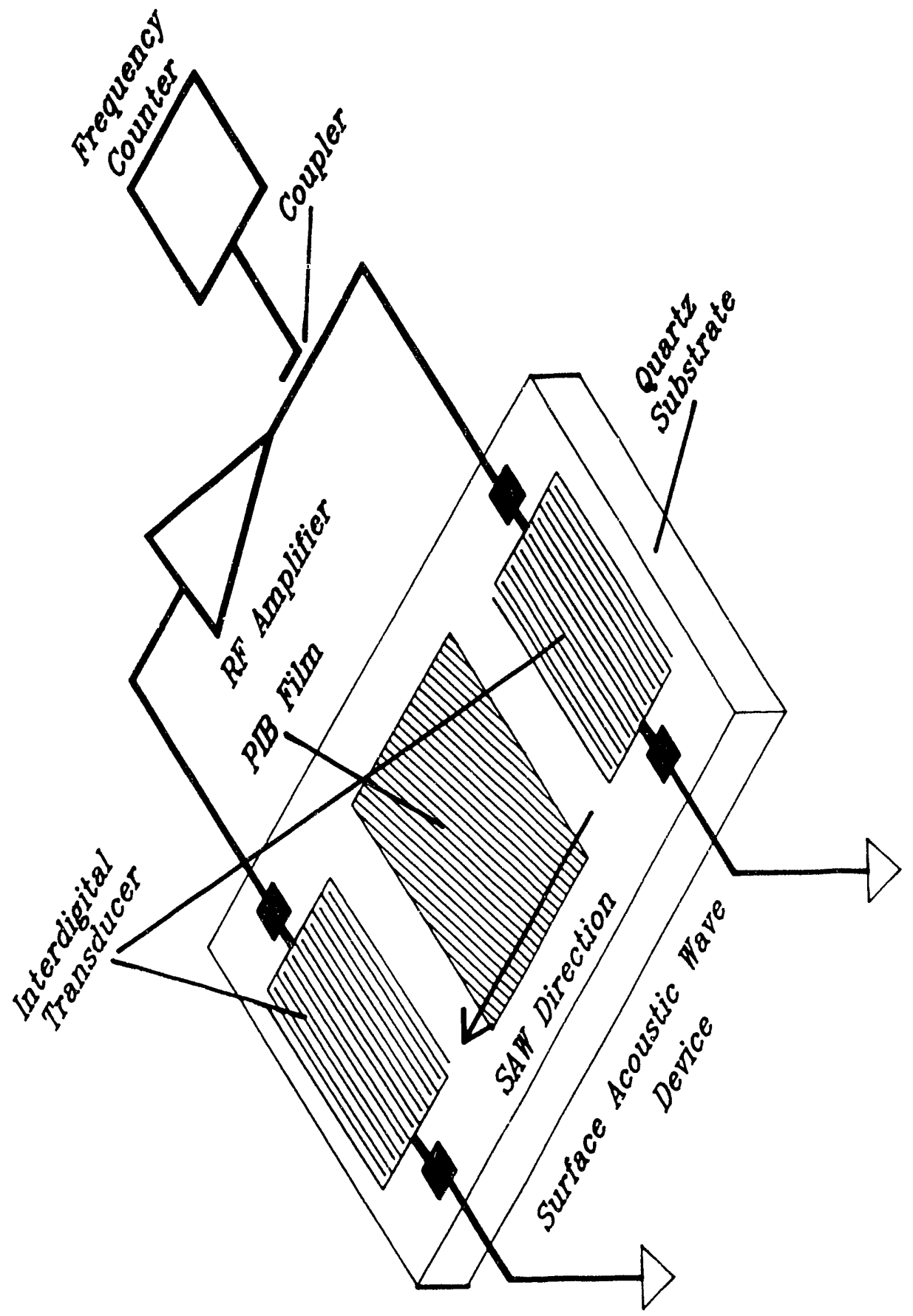

0
-1
0
0
7
0
0
0

0
0
0
4
4
5
o

感

$E$
0
0
0
0
$0-1$
0
0

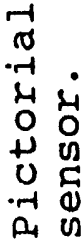

O

ن烍

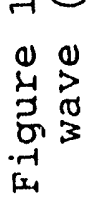


preventing damage to the sAW device and its chemically and temperature sensitive film.

Preliminary tests of the device on $\mathrm{CCl}_{4}$ in the synthetica quartz reactor (a small scale version of the MBEv) indicated that the device responds linearly to $\mathrm{CCl}_{4}$ in a background atmosphere of steam and $\mathrm{CO}_{2}$ with a proportionality constant of $1.6 \mathrm{~Hz} / \mathrm{ppm}$. It appears that the sensor can monitor the destruction of vocs $\left(\mathrm{CCl}_{4}\right)$ with little interference from the other reaction products (steam and $\mathrm{HCl}$ ), and has a minimum sensitivity (detection limit) on the order of $200 \mathrm{ppm} \mathrm{CCl}_{4}$.

The remainder of this report outlines the details of the design and testing of the SAW sensor. Section 2 gives a detailed overview of the physical description of SAW devices and their application to chemical sensing. Sections 3 and 4 deal with the design of the prototype SAW sensor system and the laboratory testing of this system at Sandia National Laboratories (SNL). section 5 describes the tests of the sensor system mounted on the synthetica steam reforming equipment, while section 6 presents some important concluding points and provides some suggestions for future studies.

\subsection{SURFACE ACOUSTIC WAVE SENSORS}

The technology used to implement a $\mathrm{CCl}_{4}$ sensor into the synthetica process involves the application of a thin, chemically-sensitive polymer film to the surface of a surface acoustic wave (SAW) device. The SAW device (Figure 1.1) consists 
of a quartz $\left(\mathrm{SiO}_{2}\right)$ substrate with two sets of interdigital transducers (IDTS) on either end of the substrate; interdigital transducers consist of a series of conductors (fingers) alternately placed on the quartz with a center-to-center spacing d. If an electric field is placed across one of the sets of IDTs, a strain is induced in the surface of the quartz as a result of its piezoelectric nature. In addition, if the electric field is oscillating at frequency $\nu$, then an oscillating strain field is generated at the surface, giving rise to a surface acoustic wave that propagates across the surface of the quartz. The acoustic wave induces a potential on the output transducer which is amplified and fed back into the first transducer, resulting in an oscillation whose frequency is determined by the physical properties of the transducer and substrate. The wavelength ( $\lambda$ ) most efficiently excited by the transducer is determined by the periodicity of the transducer finger spacing d: $\lambda=d$. The synchronous frequency $(\nu)$ at which the transducers operate most efficiently is related to the wavelength and velocity ( $v$ ) by:

$$
\nu=\frac{v}{d} \text {. }
$$

From Equation 2.1, it is clear that a change in the velocity of the wave will result in a corresponding change in the frequency of oscillation of the circuit: 


$$
\frac{\Delta v}{v_{0}}=\frac{\Delta v}{v_{0}},
$$

where $\nu_{0}$ and $v_{0}$ are the initial SAW frequency and wave velocity, respectively.

Application to the quartz surface of a thin, sensitive film capable of binding species (from the gas phase) and changing the wave velocity of the quartz surface of the sAw device results in a system capable of sensing. In the system described herein, a thin polyisobutylene (PIB) film with a thickness of $1800 \AA$ is spin cast onto a SAW device. The system detects the presence of an organic vapor when the vapor is physisorbed by the thin film, because the resulting mass and viscoelasticity changes modify the velocity of wave propagation on the quartz surface and result in a measurable and reproducible frequency shift. The following sections contain a detailed explanation of the fundamentals of SAW behavior and can be skipped.

\subsection{PERTURBATIONS IN ACOUSTIC WAVE PROPAGATION}

In acoustic wave sensor applications, the measurand is typically a change in wave velocity $v$ and/or attenuation $\alpha$ induced by the interaction of the device with its environment. consequently, it is important to understand how these interactions cause a sensor response. Changes in wave velocity and attenuation are fundamentally related to changes in wave energy density and power dissipation. With regard to velocity changes, the power density P (power/area) carried by a wave can 
be related to the wave energy density $U$ (energy/volume) stored in a lossless medium. Considering a unit cube through which a wave is passing, the transit time for the wave across the cube is $\tau=$ $1 / \mathrm{v}$. When the wave passes through the cube, the energy density in the cube increases by the incident power times the transit time: $\mathrm{U}=\mathrm{Pr}=\mathrm{P} / \mathrm{V}$. Thus,

$$
\mathrm{P}=\mathrm{UV}
$$

This relation can be used to relate changes in wave energy density to changes in wave velocity in a lossless medium, i.e., one in which $\mathrm{P}$ is constant. Differentiating Equation 2.3 yields

$$
\frac{\Delta v}{v_{0}}=-\frac{\Delta U}{U_{0}}
$$

where $v_{0}$ and $U_{0}$ denote unperturbed propagation velocity and energy density, respectively. This represents a fundamental relation between wave velocity and energy density: the fractional change in wave velocity is proportional to minus the fractional change in wave energy density. Thus, changes to the medium that affect the wave energy density change the wave velocity.

The peak kinetic energy density $U_{K}$ for a solid is given by:

$$
\mathrm{U}_{\mathrm{K}}=\frac{1}{2} \rho \sum_{\mathrm{i}=1}^{3} \dot{\mathrm{u}}^{2} \mathrm{i}
$$

where $\rho$ is the mass density of the medium and $u_{i}$ is the 
displacement of the medium along the ith dimension. Implicit differentiation of the varying quantities leads to

$$
\frac{\Delta \mathrm{U}_{\mathrm{K}}}{\mathrm{U}_{\mathrm{O}}}=\frac{\Delta \rho}{\rho_{\mathrm{O}}} .
$$

Thus, from Equation 2.4, the fractional change in wave velocity is minus the fractional change in mass density of the medium:

$$
\frac{\Delta \nu}{\nu_{0}}=-\frac{\Delta \rho}{\rho_{0}} \text {. }
$$

Thus, if a plane wave propagates in a medium in which the mass density changes, the wave velocity is shifted as indicated in Equation 2.7 .

Next, the effect on wave propagation of power dissipation in a lossy medium is considered. From conservation of energy, the power dissipated by the wave $\mathrm{P}_{\mathrm{d}}$ (power/volume) must be balanced by a reduction in power transmitted by the wave $P$. If the wave is propagating in the $x$ direction, then

$$
\mathrm{P}_{\mathrm{d}}=-\frac{\partial \mathrm{P}}{\partial \mathrm{x}}
$$

since energy density and power flow are proportional to the square of wave amplitude, in a lossy medium

$$
P(x)=P_{0} e^{-2 \alpha x},
$$


where $P_{0}$ is the input power density in the medium. Therefore,

$$
\frac{\partial \mathrm{P}}{\partial \mathrm{X}}=-2 \alpha \mathrm{P}
$$

Combining Equations 2.8 and 2.10 yields

$$
\alpha=\frac{\mathrm{P}_{\mathrm{d}}}{2 \mathrm{P}}
$$

Equation 2.11 gives the relationship between wave attenuation and power dissipation in the medium: specifically, attenuation is half the ratio of power dissipated to power transmitted by the wave. Note that in the derivations of this section, velocity and attenuation changes depend on ratios of energy and power, not on absolute levels. Consequently, in the small-signal limit, velocity and attenuation changes are independent of wave amplitude.

In general, perturbations change both energy storage and power dissipation and thus result in a combination of velocity and attenuation changes. The manner in which the propagation of the wave is described is therefore important and will be discussed here briefly.

The continuous propagation in the $x$ direction of a wave with angular frequency $\omega$ is described generally as

$$
\vec{u}(x, y, z, t)=\vec{u}(x, y, z) \text { e }(j \omega t-\gamma \gamma)
$$


where $\gamma$ is a complex propagation factor representing both attenuation and wavenumber $(k)$ :

$$
\gamma=\alpha+j k=\alpha+j \frac{\omega}{v}
$$

Changes in wave propagation can thus be represented by

$$
\Delta \gamma=\Delta \alpha+j\left[\frac{\Delta \omega}{v_{0}}-k_{0} \frac{\Delta v}{v_{0}}\right]
$$

When a SAW device is included as the feedback element in an oscillator circuit, there are two conditions that must be met for oscillation to occur. The first condition requires that the round-trip phase shift be an integral multiple of $2 \pi$ radians (boundary conditions are met). Thus, the bracketed terms in Equation 2.14 must be zero. Setting these terms to zero results in Equation 2.2 as expected. The second condition requires that the sum of all transduction and propagation losses be less than the gain in the circuit (i.e., the circuit gain is above threshold and constructive interference occurs).

$$
20 \log (\Delta \alpha L)+2 T<\text { Gain }(d B)
$$

where $L$ is the center-to-center spacing between the IDTs, and $T$ is the loss in $\mathrm{dB}$ associated with each transducer. 
The stress-free boundary imposed by the surface of a crystal gives rise to a unique acoustic mode whose propagation is confined to the surface and is therefore known as a surface acoustic wave (SAW). In 1887, Lord Rayleigh discovered this mode of propagation in which acoustic energy is confined very near the surface of an isotropic solid.4 This mode, now known as the Rayleigh wave, is of interest to seismologists because it is excited by earthquakes. The utility of Rayleigh waves in sensor applications is also due to the surface confinement of energy, allowing them to be excited by surface electrodes in piezoelectric materials and also making the wave extremely sensitive to surface perturbations.

In order to satisfy the stress-free boundary condition, coupled compressional and shear waves propagate together in a SAW such that surface traction forces are zero (i.e., $\mathbf{T} \cdot \mathbf{Y}=0$, where $\mathbf{y}$ is a unit vector normal to the device surface and $\mathbf{T}$ is the stress tensor for the medium). The generalized surface acoustic wave, propagating in the $z$-direction, generates a displacement profile $\mathbf{u}(y)$ that varies with depth $y$ into the crystal as

$$
u(x, y, z, t)=\left[u_{x}(y) e^{j \phi_{1} \vec{x}}+u_{y}(y) e^{j \phi_{2} \vec{y}}+u_{z}(y) e^{j \phi_{3} \vec{z}}\right] e^{(j \omega t-\gamma z)},
$$

where $u_{x}, u_{y}$, and $u_{z}$ represent displacement components and $\phi_{i}$ the phases of the components with respect to $u_{z}$. The terms $x, y$, and $z$ represent unit vectors in the $x, y$, and $z$ directions, 
respectively (see Figure 2.1). The component $u_{y}$ is perpendicular to the surface, $u_{z}$ is in the direction of propagation, and $u_{x}$ is transverse to the yz-plane (i.e., the sagittal plane).

The displacement components $u_{i}(y)$ vary approximately as $e^{-2 \pi y / \lambda}$, where $\lambda$ is the SAW wavelength along the surface and $y$ is the distance into the substrate; amplitude thus decays rapidly with distance into the bulk interior of the crystal (see Figure 2.1). A cross-sectional view of the strain field generated by a surface wave propagating along the surface of a crystal is shown Ln Figure 2.1. The strain energy density, also shown in the figure, indicates that the majority of wave energy is contained well within one wavelength of the surface. At higher frequencies (i.e., shorter wavelengths), acoustic energy is confined more closely to the surface and wave sensitivity to surface perturbations increases.

The sensitivity of SAW devices to surface perturbations is dependent upon the wave amplitude at the surface, which can be represented by the surface particle velocities $v_{x o}, v_{y o}$, and $v_{z o}$ in the $x_{-}, y^{-}$, and $z$-directions, respectively. For $\mathrm{ST}-\mathrm{cut}^{5}$ quartz, these values are $\mathrm{v}^{2}$ xo $=0.13 \omega \mathrm{P}, \mathrm{v}^{2}$ yo $=1.34 \omega \mathrm{P}$, and $\mathrm{V}^{2} \mathrm{z}_{\mathrm{O}}=0.88 \omega \mathrm{P}$ where $\omega \mathrm{P}$ is the product of the angular frequency and the power density.

For propagation in an isotropic medium or along a pure-mode direction of a crystal (e.g., a plane of symmetry), Equation 2.16 reduces to a Rayleigh wave, characterized by having no transverse component: $u_{x}=0$. Since $u_{y}$ and $u_{z}$ are $\pi / 2$ out of phase, the 

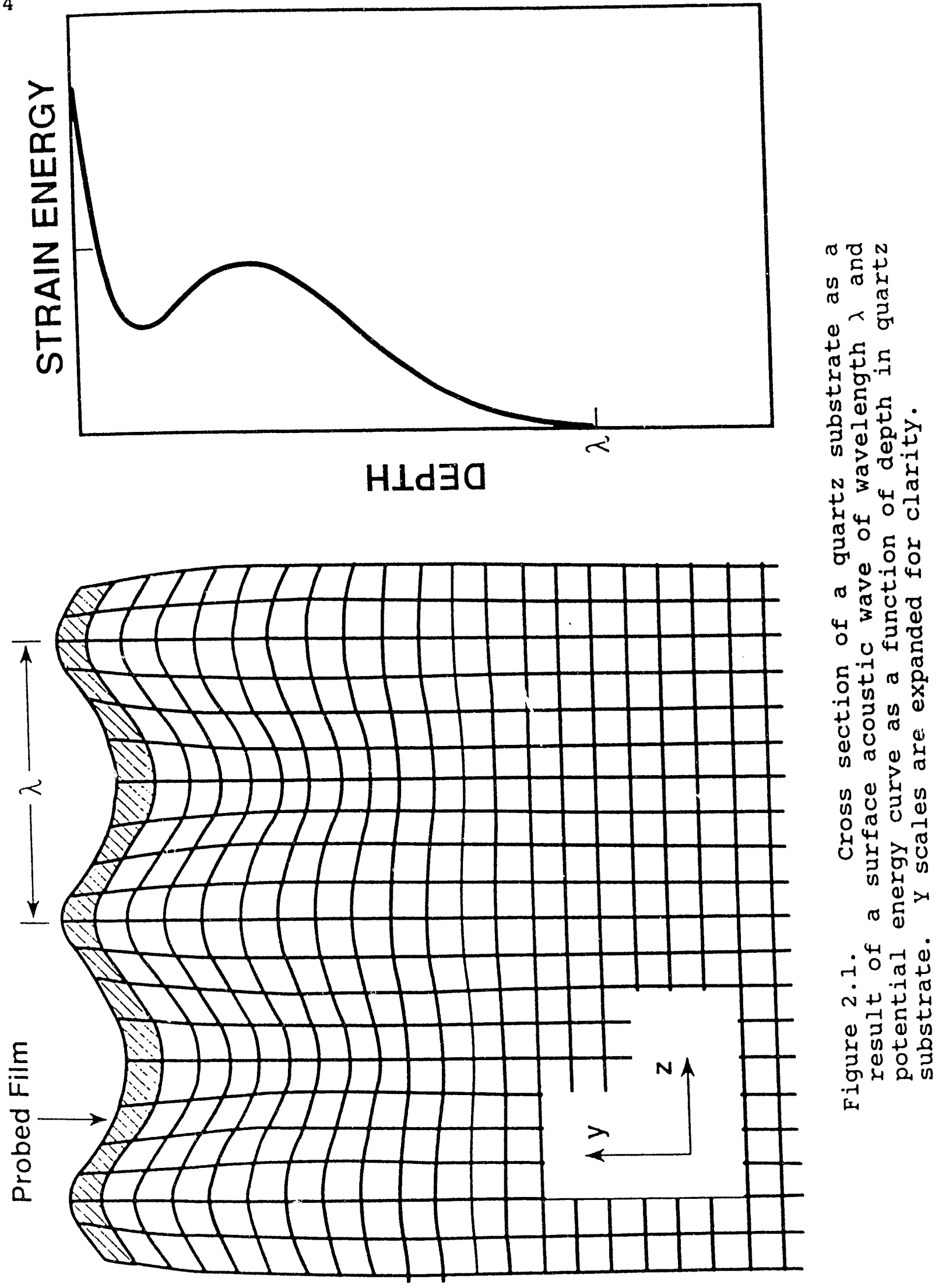

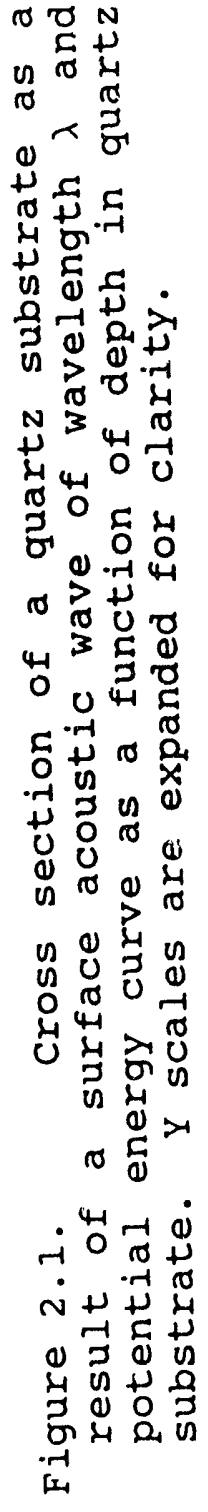


molecules of the film move in an elliptical orbit in the sagittal plane; the surface motion resembles that of the ocean under the influence of a passing wave.

The presence of the surface-rormal displacement component makes the SAW poorly suited for liquid sensing applications. When the SAW medium is contacted by a liquid, this component generates compressional waves in the liquid; the power thus dissipated leads to excessive attenuation of the SAW. 6

\subsubsection{SAW EXCITATION AND DETECTION}

The discovery, by R. M. White 4 of the University of california at Berkeley, that surface acoustic waves could be excited and detected by lithographically patterned interdigital electrodes on the surface of piezoelectric crystals has led to widespread use of SAW devices in a number of signal-processing applications. These include frequenc: filters, resonators, delay lines, convolvers, and correlators. 7,8,9

A surface acoustic wave (SAW) is most conveniently excited on a piezoelectric crystal using an interdigitated electrode pattern, or interdigital transducer (IDT), as shown in Figure 1.1. Application of a voltage between alternately connected electrodes causes a periodic electric field to be imposed on the crystal. When an alternating voltage is applied, an oscillating spatially periodic strain field is generated in the piezoelectric crystal that generates a standing surface acoustic wave. This standing wave gives rise to propagating waves that are launched 
in both directions away from the transducer; the wavefronts are parallel to the transducer fingers.

As discussed above, the transducer operates most efficiently when the SAW wavelength matches the transducer periodicity $d$ (Figure 2.2). This occurs when the transducer is excited at the synchronous frequency, defined by $\nu_{0}=v_{0} / d$, where $v_{0}$ is the SAW propagation velocity.

The propagation of the mechanical wave in a piezoelectric medium is accompanied by an associated wave potential $\Phi$. When the wave is incident on a receiving transducer, this potential induces a current flow in each transducer electrode; these currents combine to produce a current flow in the external detection circuit. The addition of current contributions in the receiving transducer is optimized when the transducer periodicity matches the acoustic wavelength. Thus, a reciprocity relation holds, as it must for a passive linear device, between the wave and external signals.

\subsubsection{INTERDIGITAL TRANSDUCER FREQUENCY RESPONSE}

Each transducer finger may be considered to be a discrete source for the generation of surface waves in a piezoelectric medium. A simple transfer function relates the continuous wave (CW) voltage $v_{1}$ applied to a finger and the electrical potential $(\Phi)$ associated with the waves radiated in each direction:

$$
\Phi=\mu_{s} v_{1}
$$




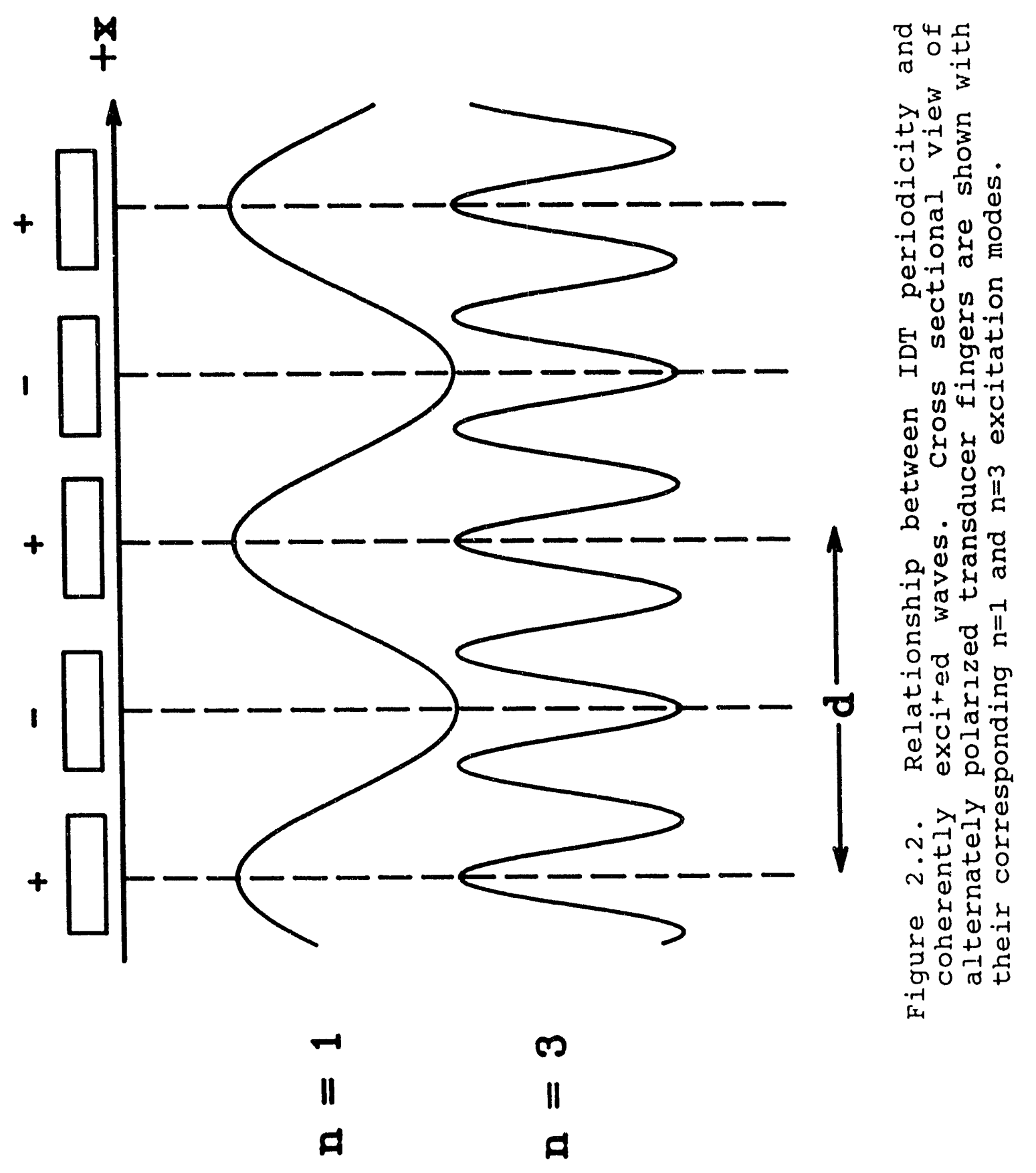


where $\mu_{S}$ is a substrate-dependent parameter, $\Phi^{+}$is associated with the rightward propagating SAW and $\Phi^{-}$with a leftward propagating SAW. The parameter $\mu_{\mathrm{s}}$ may be considered frequency independent. The frequency response of the transducer arises mainly from interference between finger contributions, and is relatively insensitive to the frequency response of the individual fingers. This approximation is typically made in analyzing wave scattering from an array of elements: the "element factor" (individual fingers) is typically considered frequency-independent when compared with the "array factor" (entire set of fingers).

When an array of fingers is excited, as occurs with an interdigital transducer (IDT), the wave potential for a rightward propagating wave $\Phi^{+}$evaluated at position $z$ is a vector sum of the contributions from each finger:

$$
\Phi^{+}(z)=\mu_{s} \sum_{n=0}^{N_{f}-1} v_{n} e^{j k\left(z-z_{n}\right)},
$$

where $z_{n}$ is the position of each finger excited with voltage $v_{n}$, and $\mathrm{N}_{f}$ is the total number of fingers. Equation 2.18 has the form of a discrete Fourier transform of the sequence $v_{n}$. consequently, the frequency response of the device is proportional to the Fourier transform of the sequence of transducer finger contributions. Schemes have been devised to 
vary the individual finger contributions in order to achieve a desired frequency response (see for example the excellent books on SAW filter design by Datta ${ }^{7}$, Morgan ${ }^{8}$, and Ristic ${ }^{9}$ ). If $\mathrm{N}_{f}$ identical fingers are spaced periodically a distance $d / 2$ apart and excited with alternating voltages $v_{n}=(-1)^{n} v_{0}$, Equation 2.18 becomes

$$
\Phi^{+}(0)=\mu_{s} v_{0} \sum_{n=0}^{N_{f}-1}(-1)^{n} e^{-(j n k d / 2)}
$$

The sum in Equation 2.19 is a geometric series whose elements become unity and add constructively when $\mathrm{kd} / 2=\mathrm{m} \pi$ ( $\mathrm{m}$ is an odd integer). This condition defines the relationship between SAW wavelength $\lambda$ and transducer periodicity $d$ for coherent addition, as shown in Figure 2.2. The IDT excites harmonics at odd multiples of the synchronous frequency: $\nu_{m}=m \nu_{1}$.

Moving away from the synchronous frequency, the addition of components from individual fingers becomes incoherent, giving rise to the frequency response

$$
\left|\Phi^{+}(f)\right|=\left|\frac{\sin (X)}{X}\right|
$$

in which

$$
\mathrm{X}=\frac{\mathrm{N}_{\mathrm{P}} \pi\left(\nu-\nu_{0}\right)}{\nu_{0}},
$$


where $\nu_{0}$ is the transducer's synchronous frequency and $N_{p}$ is the number of IDT periods: $N_{p}=N_{f} / 2$. The wave potential as a function of the detuning parameter $x$, described by Equation $2.20 \mathrm{~b}$, is shown in Figure 2.3. Note that when $\mathrm{x}$ is a multiple of $\pi, \Phi^{+}$is zero (a result of complete cancellation between finger contributions). Consequently, the frequency interval B between the first nulls on either side of the synchronols frequency is

$$
\mathrm{B}=\frac{2}{\mathrm{~N}_{\mathrm{P}}} \text {. }
$$

Thus, the transducer bandwidth is inversely proportional to the number of IDT fingers. Since a narrow bandwidth is desirable for oscillator applications in order to avoid spurious oscillations and to improve stability, a large number of fingers and a small finger spacing are desirable.

The frequency response measured between a pair of transducers having $\lambda_{0}=32 \mu \mathrm{m}$ and $\mathrm{N}_{\mathrm{p}}=50$ finger pairs is shown in Figure 2.4. The amplitude, shown on a log (decibel) scale, shows the characteristic $\sin (\mathrm{X}) / \mathrm{x}$ behavior.

\section{2 .3 SAW PERTURBATION MECHANISMS}

When SAW devices are used for sensors or thin-film characterization, the measured responses arise from perturbations in wave propagation characteristics, specifically wave velocity and attenuation, resulting from interactions between the SAW and a surface film. Because a SAW propagating in a piezoelectric 


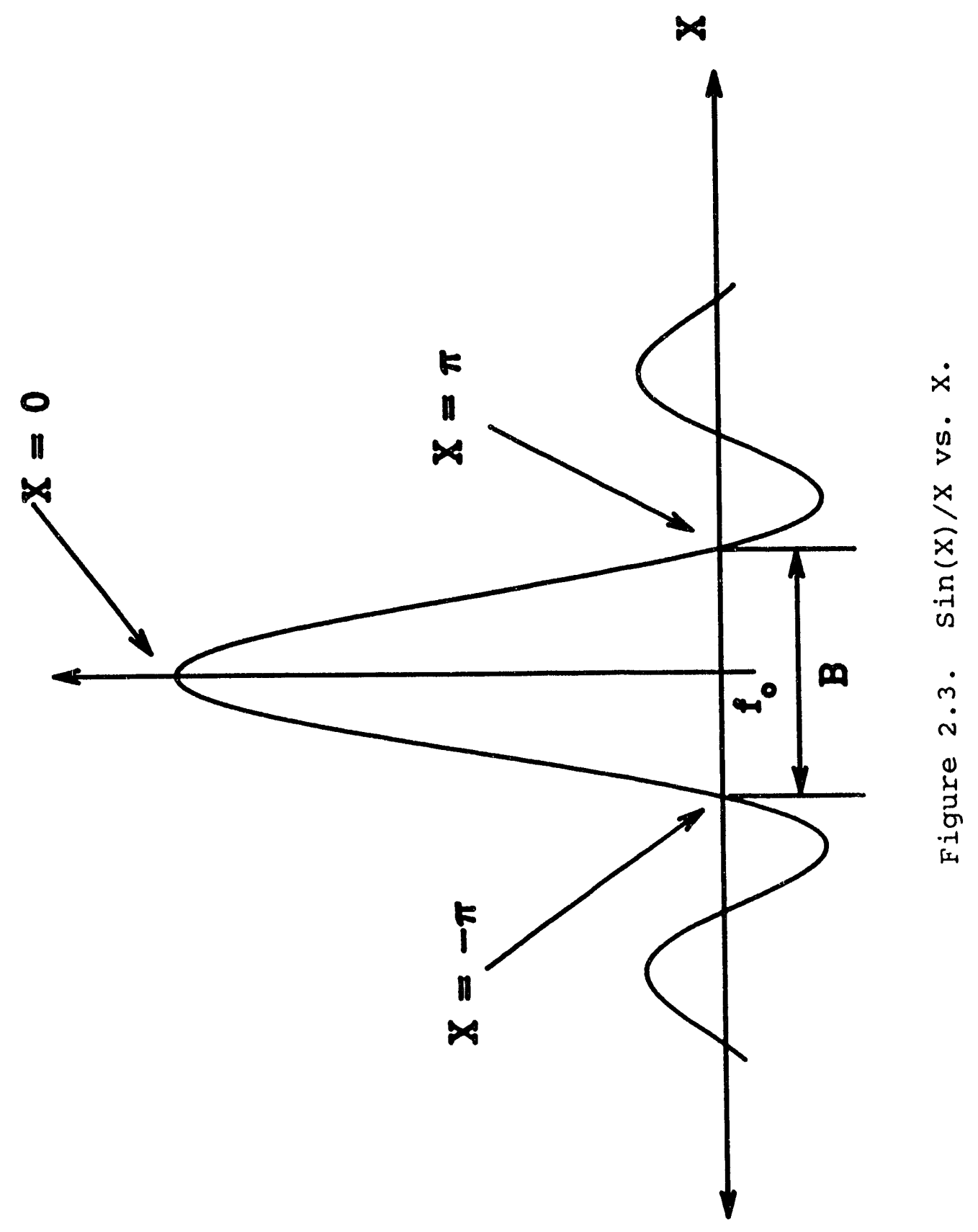




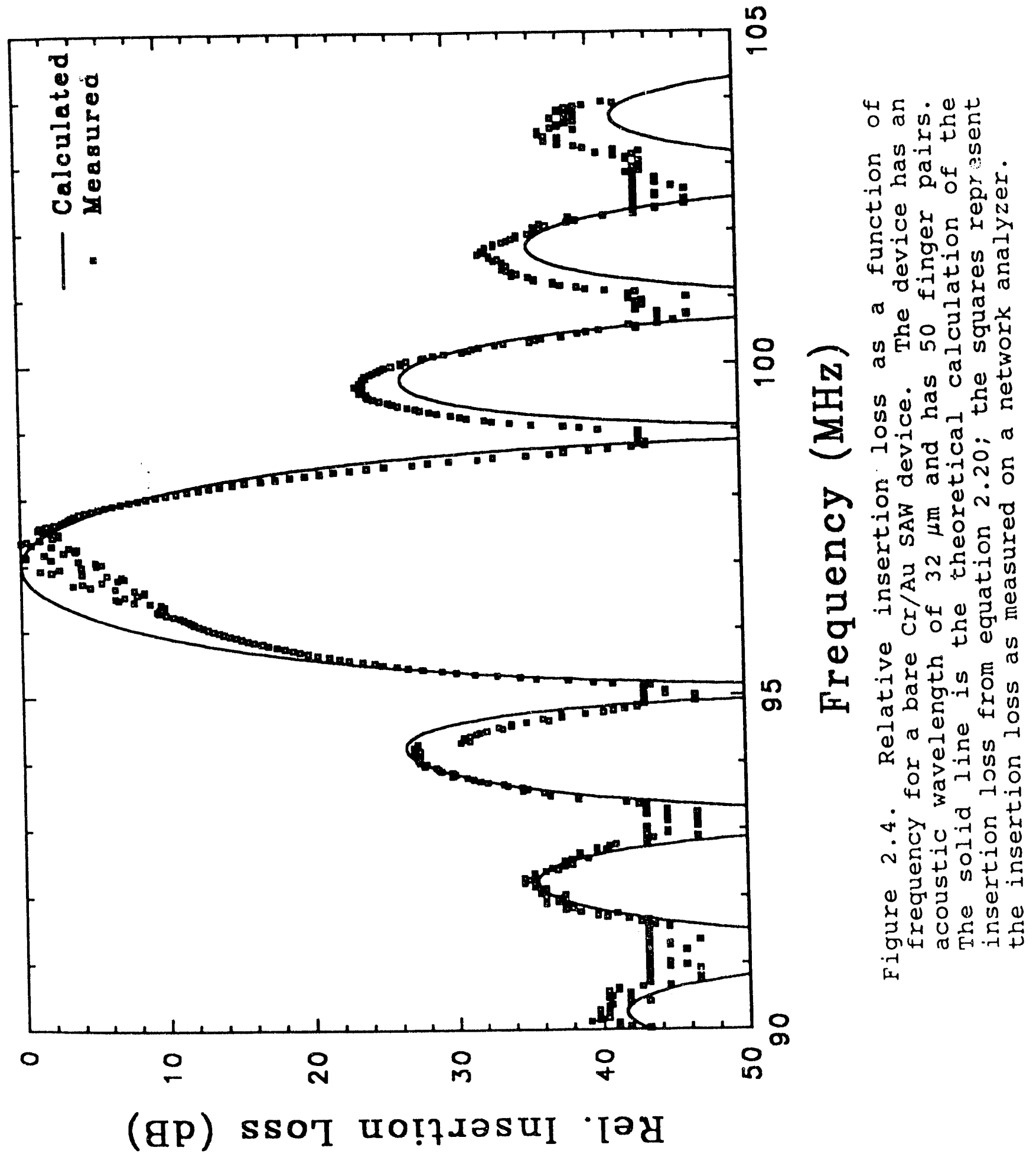


medium generates both mechanical deformation and an electrical potential, both mechanical and electrical coupling between the SAW and surface film are possible consequently, a number of interactions between surface waves and surface films have been found that give rise to velocity and attenuation responses. SAW-film interactions that arise from mechanical coup.ing between the wave and film include mas: loading caused by the translation of surface mass by the SAW suriace displacement, and elastic and viscoelastic effects caused by SAW-induced deformation of a surface film. The next section examines the velocity and attenuation changes caused by mass loading interactions between SAWs and surface films.

\subsubsection{SAW MASS LOADING}

The simplest interaction, and the one most utilized for SAW sensor applications, is the response due to changes in the mass density (mass/area) on the device surface. The harmonic motion of the crystal surface caused by the passing suriace wave causes molecules bound to the surface to move in an elliptical orbit synchronously with the SAW surface displacement. The effect on wave velocity and attenuation of this interaction may be derived from energy considerations.

synchronous movement with the surface acoustic wave of i surface film causes an increase in ths kinetic energy density $U_{K}$ of the wave without dissipating any wave energy. From the discussion in section 2.1, this is expected to change the wave 
propagation velocity without affecting attenuation. The change in average kinetic energy per area of surface is

$$
\Delta \mathrm{U}_{\mathrm{K}}=-\frac{\rho_{\mathrm{S}}}{4}\left(\mathrm{v}^{2} \mathrm{xo}_{\mathrm{O}}+\mathrm{v}^{2} \mathrm{yo}_{\mathrm{O}}+\mathrm{v}^{2} \mathrm{zo}_{\mathrm{O}}\right)
$$

where $v_{x o}$, $v_{y o}$, and $v_{z o}$ are the molecule velocities at the surface and $\rho_{s}$ is the surface mass density. Particle velocities are related to particle displacement $u$ by $v_{i}=j \omega u_{i}$. This increase in kinetic energy density results in a decrease in wave velocity, according to Equation 2.4. Combining Equations 2.3, 2.4 , and 2.22 yields an expression for the change in wave velocity arising from surface mass loading:

$$
\frac{\Delta v}{v_{0}}=-\frac{v_{0} \omega \rho s}{4}\left[\frac{v^{2} x o}{\omega P}+\frac{v^{2} y_{0}}{\omega P}+\frac{v^{2} z_{0}}{\omega P}\right] .
$$

Due to the greater confinement of wave energy near the surface that occurs as operating frequency increases, surface molecule velocities increase in proportion to $(P \omega)^{1 / 2}$. Thus, the quantities in parentheses $\left(\mathrm{v}_{10}{ }^{2} / \omega \mathrm{P}\right)$, being independent of wave amplitude and dipending only on the substrate material, remain constant. Slobodnik et al. have tabulated these normalized surface particle velocities for a large number of substrates. 10 propagation in the $x$-direction in the ST-cut of quartz (a rotated cut chosen for its desirable temperature characteristics) results in three components of particle velocity because of the lack of 
crystal symmetry. Grouping all the substrate-dependent constants together results in the expression for the mass-induced change in SAW propagation velocity:

$$
\frac{\Delta v}{v_{0}}=-c_{m}^{\nu} \rho_{s},
$$

where the mass sensitivity factor $c_{m}$ is

$$
c_{m}=\frac{2 \pi v_{0}}{4}\left[\frac{v^{2} x o}{\omega P}+\frac{v^{2} y o}{\omega P}+\frac{v^{2} z_{0}}{\omega P}\right]
$$

Note that the frequency dependence of the SAW mass sensitivity (fractional velocity change $\Delta v / v_{0}$ ) varies with operating frequency $\nu_{0}$. Because the mass layer is assumed (in this case) to be lossless, Equation 2.24 implies that attenuation is unchanged by mass loading. The assumption that the mass layer is lossless is not valid for many practical applications; however, the effect on the mass sensitivity is typically small and can generally be neglected. For lossy materials, additional interaction mechanisms such as viscoelasticity arise making the SAW response no longer due simply to mass loading.

The velocity and attenuation changes resulting from depositing a gold layer on a 97-MHz SAW device using an ST-cut quartz substrate are shown in Figure 2.5. Velocity decreases linearly in this thickness regime, yielding $c_{m}=1.32 \times 10^{-6}$ $\mathrm{cm}^{2}-\mathrm{s} / \mathrm{g}$, in good agreement with the mass sensitivity factor calculated above for a $100 \mathrm{MHz}$ SAW. The figure demonstrates 
(udd) $y / 20$

䟺

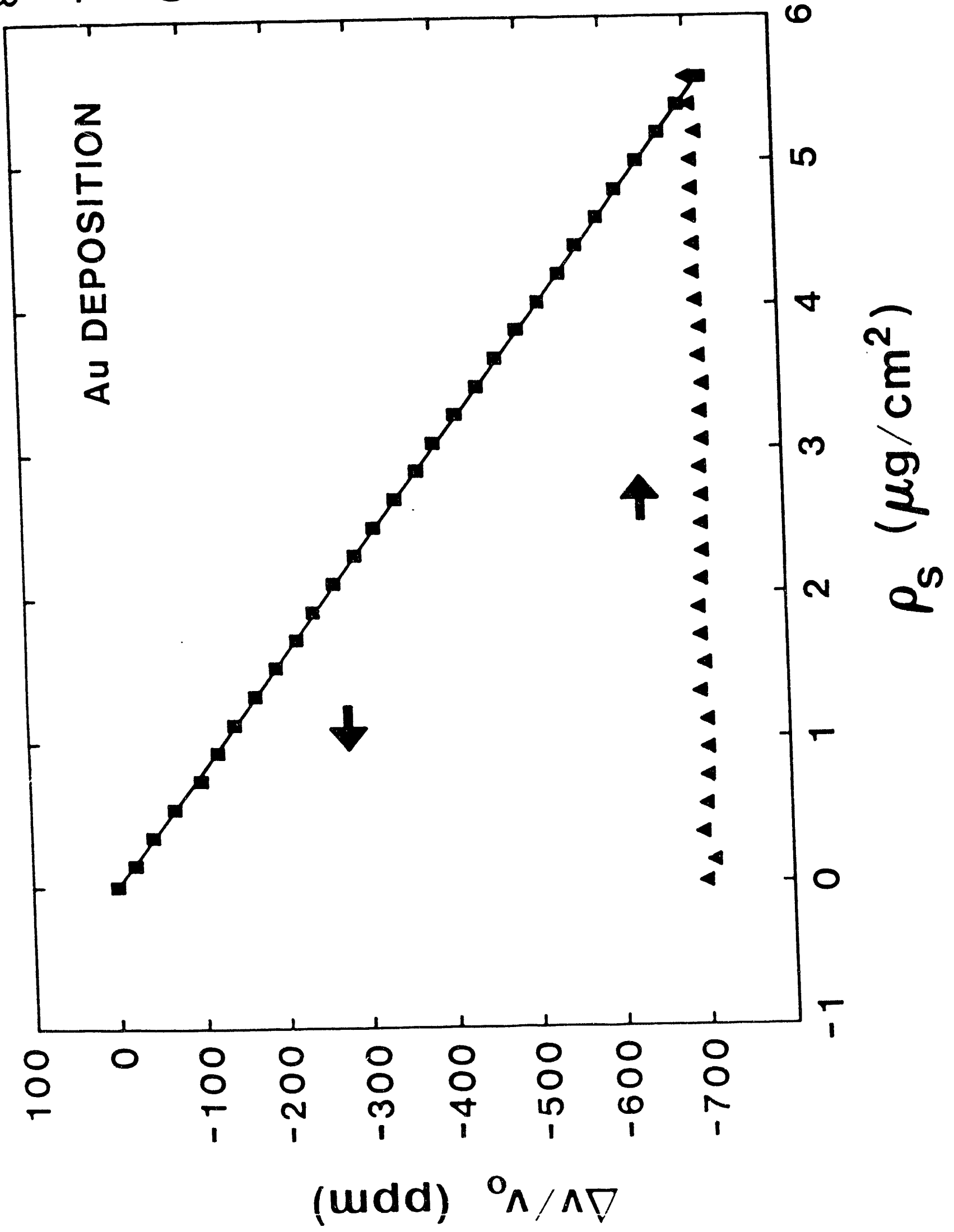

ช) U 07

N 0 N $N$

$\rightarrow E \cdot-1$ U

-1

亘

4 o d

0 出 0 U

ᄃ

(1) is

$\begin{array}{lll}0 & 0 & 0 \\ 4 & 0 & 0\end{array}$

$20+4$

둥

0 o

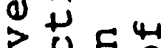

$>0 E^{4} 0$

3 ह

. ${ }^{4-1} \dot{0}$

+ 00

n $0+5$

o n

ก

$\omega>0 \quad$ n

$U>\infty$

为

म + के वे

4 $14 \leq$

म 4 C i 4.0 م - ये N山

a) 0 .

4 Un 4

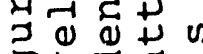
or $>$ क 
that, as predicted from the model, the relative attenuation change $(\Delta \alpha / k)$ due to mass loading is negligible in comparison with $\Delta v / v_{0}$ (shown on the same scale).

\subsubsection{SUMMARY OF SAW PROPERTIES}

In the above sections, the basic SAW sensor was described including the relationship between the change in velocity of the wave and the change in frequency. Next, a more detailed mathematical model of SAW propagation was given to demonstrate how an increase in mass density will result in a decrease in wave velocity. These arguments were expanded to a lossy medium and it was shown that the same relationship between change in frequency and change in wave velocity (Equation 2.2) holds for a lossy medium. The nature of the interdigital transducers were discussed including the frequency response and bandwidth of the devices.

Finally, it was shown how mass loading of a film on a surface acoustic wave device changes the velocity of the wave, and that the change in velocity is independent of the amplitude of the wave. A relationship between the mass sensitivity of the SAW device and its frequency and material properties was derived. The next section describes a system design that implements these SAW properties in a sensor.

\subsection{SYSTEM DESIGN}

In this section, the design of the sensor system is 
discussed, including the construction of the SAW oscillator board and the SAW test cases. In addition, the designs of the mixer and signal processing circuitry and the heater controller circuitry are presented.

The system consists of two identical SAW oscillator circuits (Figure 3.1). The SAW device of one of the oscillator circuits is open to the atmosphere via a diffusion tube and is referred to as the sensor oscillator. The SAW device of the other oscillator circuit (reference) is identical to the first except that it is isolated from the atmosphere (sealed in air). The reference circuit allows for the correction of common-mode noise signals such as temperature fluctuations.

The output of the reference and signal oscillators are mixed and the difference frequency is converted to a TTL-compatible signal by the mixer/signal processing section of the system. The TTL output frequency of the circuit is measured with the frequency counter which in turn is interfaced via the RS-232 bus with a computer.

In addition, the temperature of each individual SAW device is controlled by an operational amplifier (op amp) based temperature controller. The temperature of the sensor SAW device is more strongly coupled to the environment than that of the reference SAW device. Therefore, the temperature set point of the reference SAW device is controlled by the temperature of the sensor SAW device rather than being set to a fixed temperature.

Finally, the entire system's temperature is controlled via 

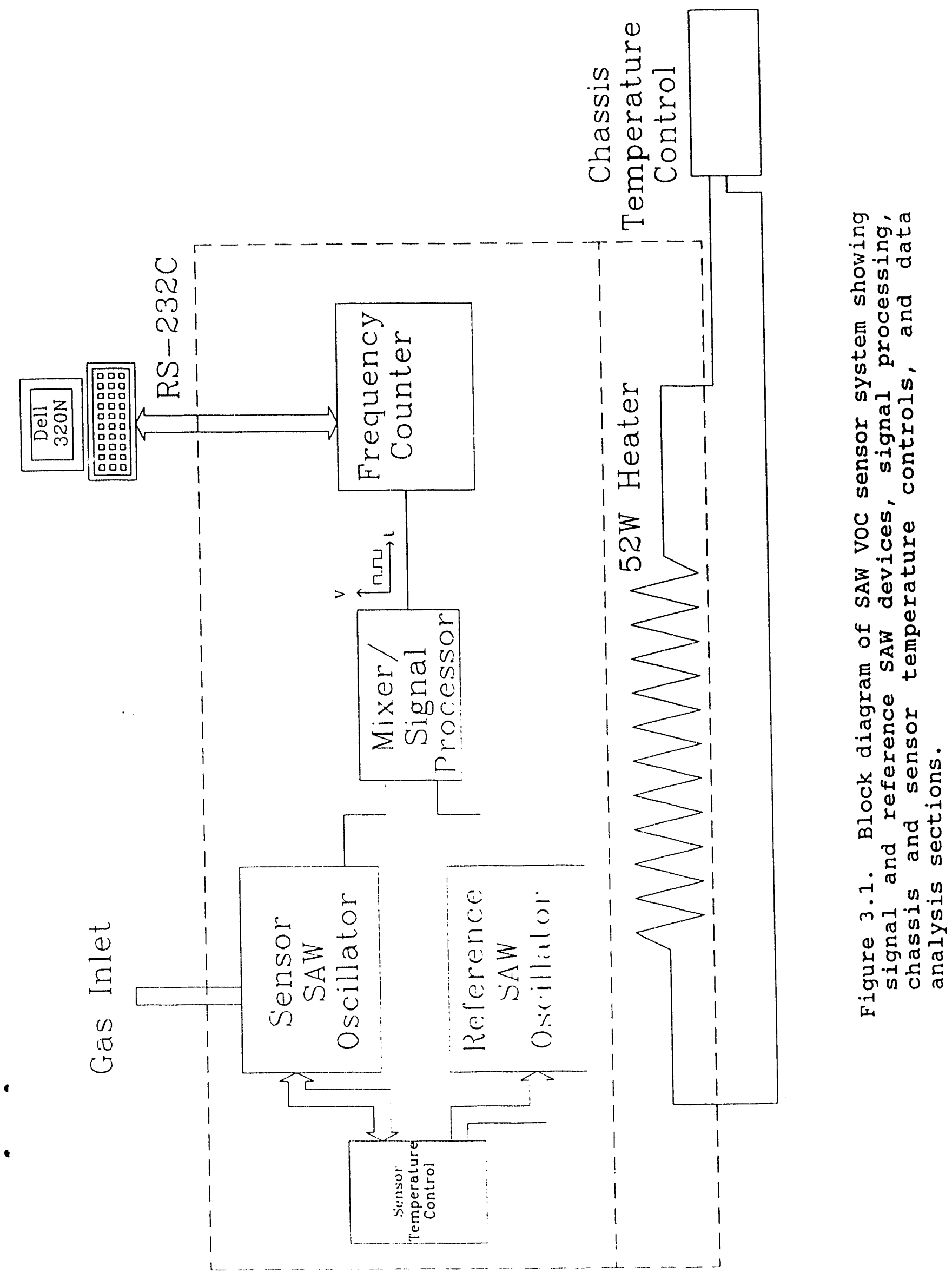
the chassis temperature controller. The chassis temperature controller consists of an omega Model CN9000 miniature controller coupled to a $52 \mathrm{w}, 120 \mathrm{~V}$ heating element mounted to the chassis.11 The system temperature controller can maintain the chassis temperature to within approximately $1^{\circ} \mathrm{C}$; the chassis controller can compensate for gross changes in the room temperature, while the individual SAW temperature controllers compensate for fine $\left(<1^{\circ} \mathrm{C}\right)$ temperature fluctuations in the SAW devices.

\subsection{SAW OSCILLATOR CIRCUIT}

The SAW oscillator circuits consist of a SAW device followed by a Mini-Circuits Laboratories PSC-2-1 3-dB splitter (Figure 3.2) that was placed on the circuit board to provide a measurement of the output power from the SAW device.12 It, along with another splitter on the input to the SAW device, allows for measurement of the attenuation change in a film due to exposure to the chemical environment. Chemical discrimination techniques employing measurement of both frequency shift and attenuation change have been demonstrated ${ }^{3}$; however, this feature was not used in this prototype.

The other output of the splitter $($ Pin-6) is input to a current-controlied attenuator (PAS-3) that is included in the circuit to allow adjustment of the oscillation threshold of the circuit. 12 By increasing the attenuation, the circuit can be made to oscillate in only the most efficient mode of the SAW 


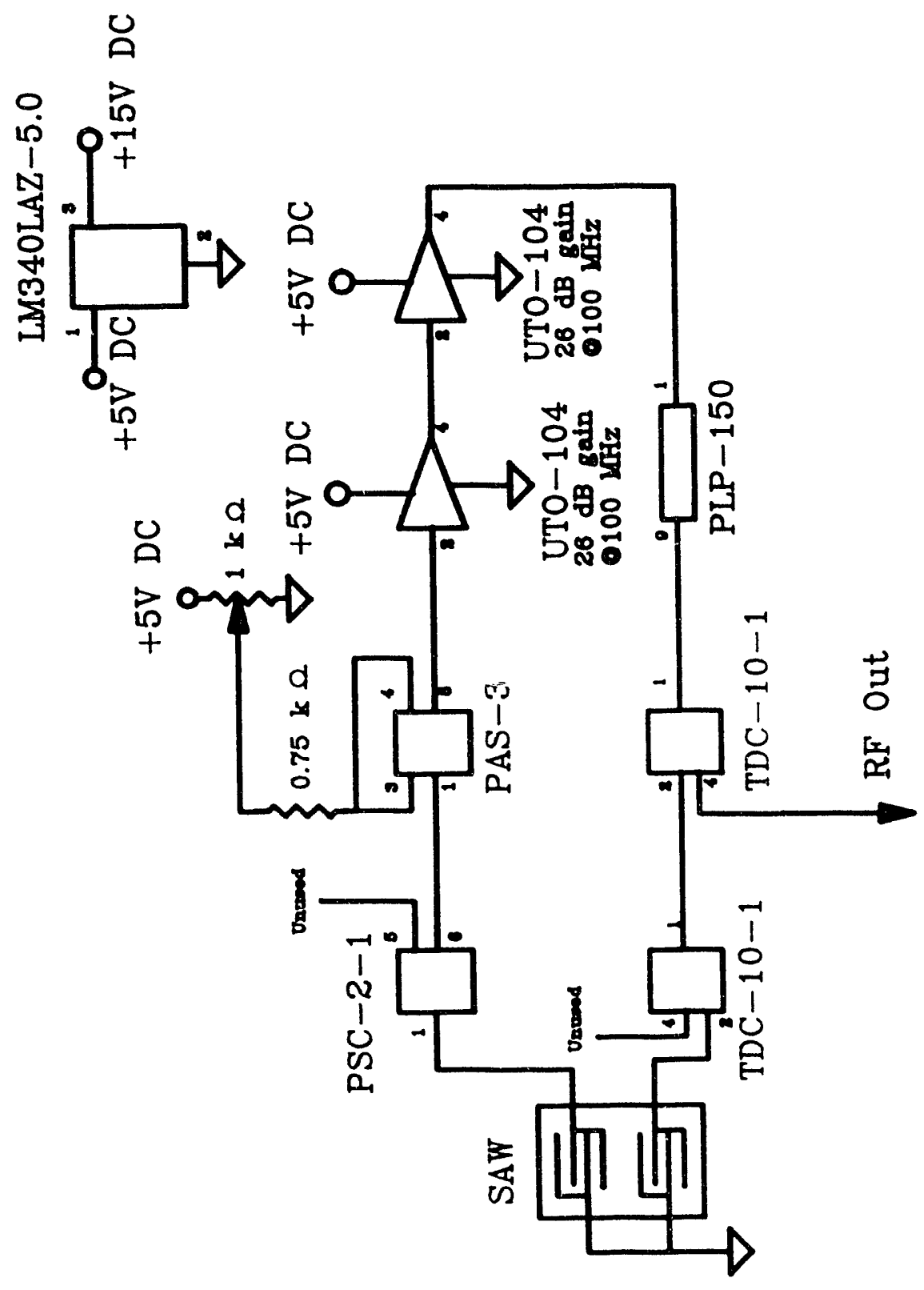

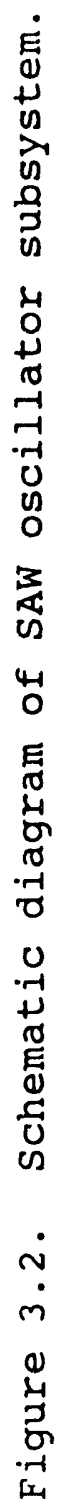


device, resulting in a much lower noise level.

The signal from the attenuator is amplified by two radio-frequency (RF) amplifiers in series (UTO-104 and UTO-250) that provide $24 \mathrm{~dB}$ of gain and $30 \mathrm{~dB}$ of gain, respectively, at saturation. 13 The output from the second amplifier is fed into a low-pass filter (PLP-150) that has a $3-\mathrm{dB}$ roll-off at $150 \mathrm{MHz}$ and is included in the circuit to eliminate harmonics generated by the saturated amplifier. 12 The signal is next coupled to a 10-dB splitter (TDC-10-1) that is used to couple a portion of the RF signal out of the circuit to the mixer processor system. Finally, the remaining signal is coupled back to the SAW device, completing the oscillator loop. This circuit was constructed on a $2.75^{\prime \prime}$ by $3.75^{\prime \prime}$ printed circuit board.

\subsection{SAW CASES}

The SAW cases designed for this project had to meet several distinct requirements. First, they had to fit on a $1.25 " \times 0.75 "$ area of the circuit board; this restriction arose because circuit boards that had been designed for a separate program were being used. At the time this project was initiated, these boards were designed to have SMA connections to connect the circuit to a separate SAW device test case. Second, they had to allow easy device replacement in the field. The second objective was achieved using spring-loaded electrical contacts built into the SAW test case. In previous SAW case designs, SAW devices were wire bonded to the contacts in the case, making replacement 
in the field impractical. Finally, since the SAW cases are exposed to a chemically harsh environment, they were constructed from corrosion resistant 300-series stainless steel.

The SAW device cases were constructed (as depicted in Figure 3.3) from a stainless steel block with a rectangular depression milled in the center to contain the SAW device. Appendix A, Figures A.1, A.2, A.3, and A.4 show mechanical drawings of the SAW case. There are four holes in the bottom of the depression through which spring-loaded electrical connectors protrude. Two of the holes are 0.0625" in diameter and two are 0.045" in diameter. The smaller holes are designed for a press fit of the Augat, Inc. spring-loaded "pogo" contacts ( $\mathrm{P} / \mathrm{N} \mathrm{C}-\mathrm{S}-\mathrm{R}) .14$ The larger holes are designed for a press fit of the pogo contacts with a $0.009 "-t h i c k$ cylindrical Teflon bushing that electrically insulates the pins from the case. The larger holes provide the RF signal input and output to the SAW devices while the smaller holes provide circuit ground connections.

The top of the case has a 1/16" o-ring groove that forms a gas-tight seal when the lid is attached. In addition, the pogo contacts are placed at a depth that allows the SAW device to be positively located by the walls of the depression when the lid is removed from the case. This requires that the $0.025^{\prime \prime}$ thick SAW device extend $0.010 "$ below the surface of the case when the lid is off of the case and the pogo contacts are not compressed. When the lid is placed on the case and the back of the device is flush with the top of the case, the SAW device is forced down 


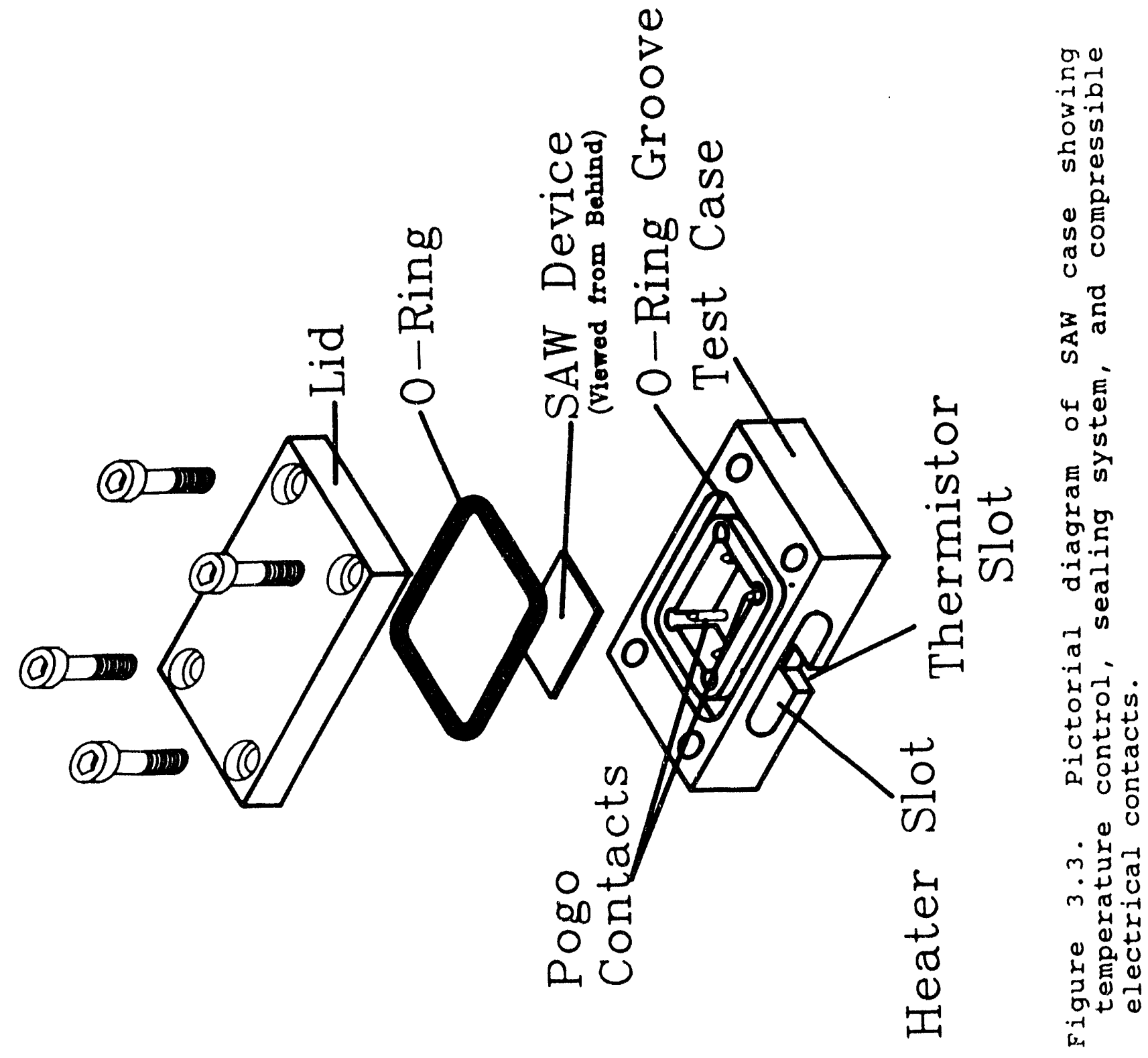


0.015". The pogo contacts are compressed to approximately half of their range of motion, making excellent contact with the $0.071 "$ square bond pad of the SAW device.

Temperature control of the SAW case is accomplished by glueing a heater resistor in the $\left(0.590^{\prime \prime} \times 0.150 " \times 0.150 "\right)$ depression milled into the side of the case. In addition, a cut $\left(0.125^{\prime \prime} \times 0.125^{\prime \prime} \times 0.063^{\prime \prime}\right)$ is milled just below the heater resistor depression to provide room for a thermistor to measure the case temperature.

There are two variations of the SAW case used in the sensor system. The first variation is hermetically sealed to contain the reference SAW device. The second variation has a $1 / 8$ " $x$ 1" stainless steel inlet tube welded to the bottom of the depression to provide a path for the gas sample to diffuse into the sensor SAW device.

\subsection{MIXER SIGNAL PROCESSOR CIRCUIT}

The output of each SAW oscillator (Pin 4 TDC-10-1) is a sinusoidal signal with an amplitude of approximately $100 \mathrm{mV}$ (peak-to-peak) and a frequency of approximately $97 \mathrm{MHz}$. The outputs are mixed by an Anzac MD-123 mixer (see Figure 3.4) and then "squared" (for TTL counter compatibility) with a comparator circuit. 15 The MD-123 is a double-balanced mixer with a $10 \mathrm{MHz}$ to $3 \mathrm{GHz}$ RF bandwidth. The mixer IF (intermediate frequency) bandwidth is DC to $3 \mathrm{GHz}$; it provides $20 \mathrm{~dB}$ of isolation between the RF inputs and the IF output in the frequency range of 


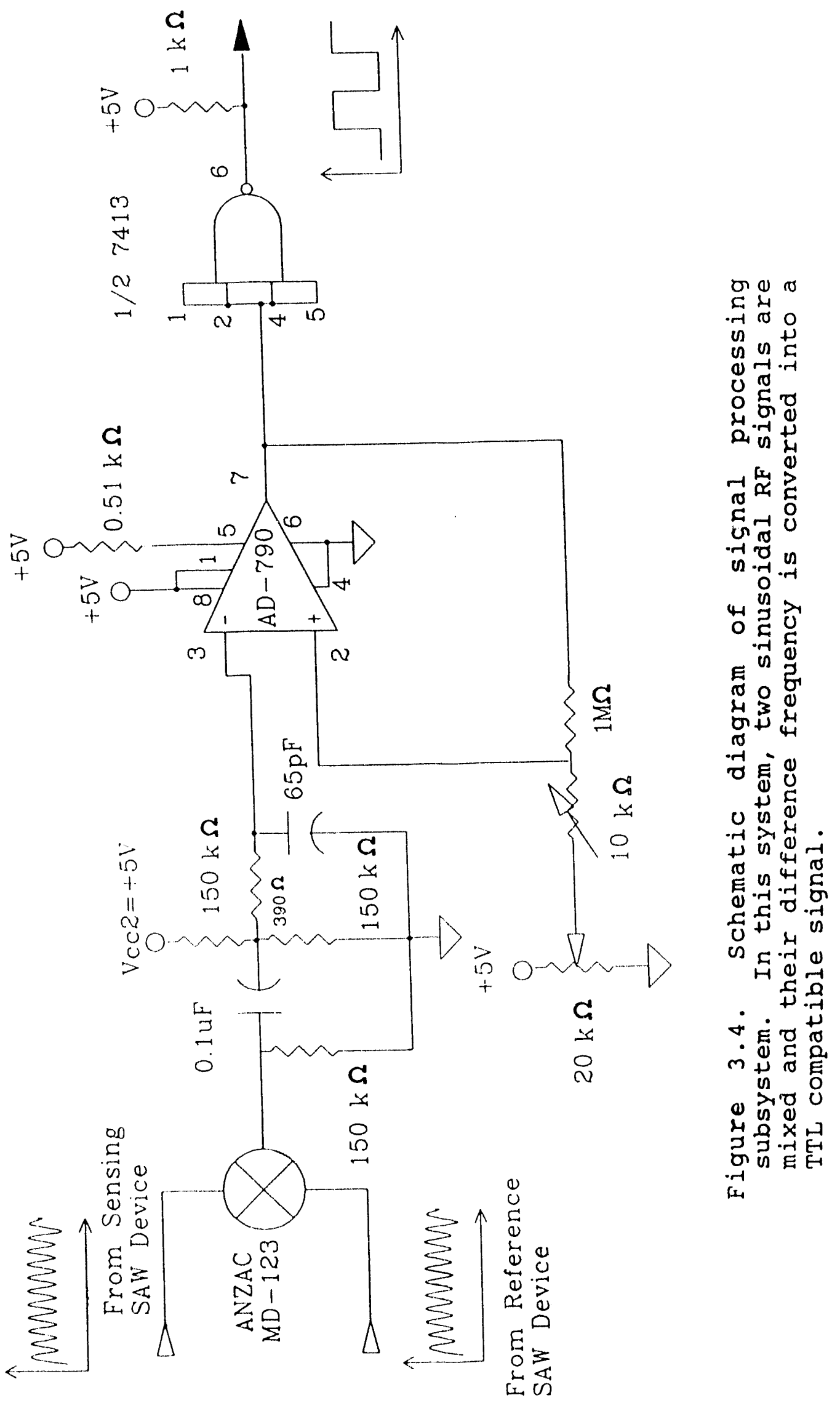


interest. The output of the mixer is capacitively coupled through a $0.1 \mu F$ capacitor to a sianal processing circuit consisting of an Analog Devices AD-790 high speed comparator and assoclated hardware. 16 The input signal is biased at $2.5 \mathrm{~V}$ by the divider circuit $(150 \mathrm{k} \Omega$ resistors) and coupled to a $390 \Omega$ resistor. The $390 \Omega$ resistor, along with a $65 \mathrm{pF}$ capacitor, form a low-pass filter with a $3-d B$ cut off at approximately $6.0 \mathrm{MHz}$; this effectively passes the difference frequency while removing the sum-frequency signal at $194 \mathrm{MHz}$. This signal is then coupled into the inverting input of an $\mathrm{AD}-790$ high-speed comparator that converts the difference signal. to a square-wave signal with the same frequency as the input sine wave. The $A D-790$ is wired to have an adjustable trigqer threshold (20 $\mathrm{k} \Omega$ variable resistor) and adjustable hysteresis (10 $\mathrm{k} \Omega$ variable resistor). The output of the comparator is input to a signetics 7413 four-input schmitt trigger to buffer the output of the comparator and provide a signal that switches between 0 and $5 \mathrm{~V}$ (TTL). 17

The TTL signal is then fed into a Newport Electronics Model P6430 TTL frequency counter (Figure 3.1) whose bandwidth is $7 \mathrm{MHz}$ and has 6 digits of output resolution. 18 Thus, for a difference signal that is less that $1 \mathrm{MHz}$, the output resolution is on the order of $1 \mathrm{~Hz}$. In addition, the $\mathrm{P} 6430$ is interfaced via RS-232C to a Dell $320 \mathrm{~N}$ notebook (PC-compatible) computer that reads the difference frequency and plots it as a function of time. 19

\subsection{HEATER CIRCUITS}


In order to keep liquid water from condensing on the sensing SAW device, it is necessary to elevate its temperature to a temperature above that of the input atmosphere - approximately $38^{\circ} \mathrm{C}$ at the sensor end of the diffusion tube. This was accomplished with the circuit shown in Figure 3.5. A heater resistor is imbedded in the SAW case and the temperature of the case is controlled by a LM255 op amp circuit. 20 The temperature of the reference SAW case is also elevated to the same temperature as the sensing SAW case to reduce temperature-induced noise in the system.

The sensing SAW case is strongly coupled to the outside environment; thus, it is more susceptible to variations in the temperature of the outside environment. The temperature control circuit was constructed to behave as a master temperature controller for the signal SAW case and a slave temperature controller for the reference SAW case. The master/slave arrangement allows the reference temperature control circuit to have a variable set point that is electronically controlled by the temperature of the sensing SAW case.

To understand the operation of the temperature control system, it is necessary to explain the master temperature controller. As indicated in Figure 3.5, the master (sensor) temperature controller consists of a resistive bridge circuit connected to an LM224 op amp. 20 The output of the op amp drives an npn power transistor that in turn controls current through the heater resistor. 


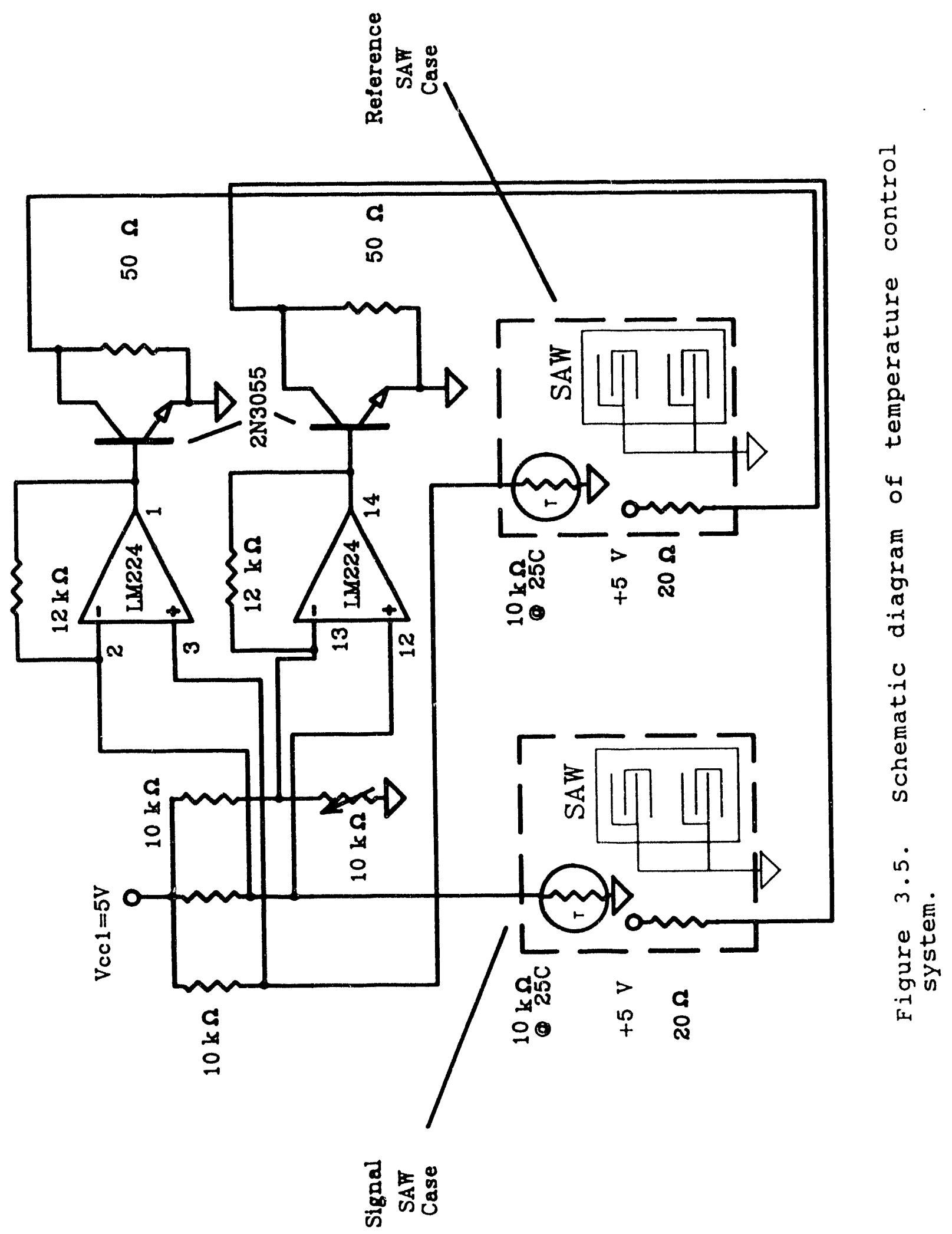


The LM2 24 op amp is designed to operate from a single-sided power supply $(+5 \mathrm{~V})$ and can only output positive voltage values. The op amp will output a non-zero voltage when the non-inverting input voltage ( $\mathrm{Pin} 3$ ) is larger than the inverting input voltage ( $\operatorname{Pin} 2)$.

The resistance bridge consists of two metal film $10 \mathrm{k} \Omega$ resistors, one $20 \mathrm{k} \Omega$ variable resistor, and a negative temperature-coefficient thermistor that is $10 \mathrm{k} \Omega$ at $25^{\circ} \mathrm{C}$. When the bridge is balanced, the voltages at the inverting and non-inverting inputs of the op amp are equal and the output of the op amp is zero. However, when the temperature of the SAW case drops below the set point (i.e., the thermistor resistance rises above the variable resistance value), the voltage at $\mathrm{Pin} 13$ becomes larger than the voltage at Pin 12, turning on the op amp and causing current flow through the heater resistor. Dissipated heat flows into the SAW case and raises the temperature of the SAW case back to the set point. The $12 \mathrm{k} \Omega$ feedback resistor on the op amp decreases the slope of the response of the op amp, reducing thermal oscillation of the SAW case.

The temperature controller for the reference SAW case works similarly to the temperature controller for the signal SAW case. However, instead of the temperature being set independently by the variable resistor, the temperature of the reference SAW case is set by the temperature of the signal SAW case. This is done by replacing the variable resistor arm of the resistance bridge with the thermistor arm of the signal 
SAW case control circuit as is illustrated in Figure 3.5. If the input gas to the signal SAW case is hot enough to heat the SAW case, the temperature of the reference SAW case will also rise automatically. Since the temperature coefficient of each SAW device is similar, each SAW oscillator changes in frequency by a similar amount. Thus, the difference frequency will remain relatively unchanged.

In the previous sections, the SAW sensing system was described in detail. This included discussion of the oscillator and signal processing section of the circuit, mechanical design of the SAW cases, and the master/slave temperature control circuits. In the next sections, the results of the laboratory and field demonstrations of the system will be discussed.

\subsection{LABORATORY TESTS}

In this section, the laboratory tests of the sensor are discussed. First, the coating of the devices are described, and studies of the short-term and long-term noise in the system, including the temperature response, are reported. In addition, the response as a function of $\mathrm{CCl}_{4}$ concentration is described and the minimum detectable limit is determined.

\subsection{POLYISOBUTYLENE FILMS}

The coating of the SAW devices was accomplished as follows. First, $0.90 \mathrm{~g}$ of polyisobutylene (PIB) was dissolved in $44.1 \mathrm{~g}$ of chloroform $\left(\mathrm{CHCl}_{3}\right)$ to obtain a $2 \%$-by-weight solution of PIB; 
the mixture of chloroform and PIB was placed in an ultrasonic cleaner to dissolve the PIB. The bare SAW devices were cleaned in boiling acetone, methanol, and isopropanol to remove any residual photoresist and other surface contaminants. Finally, the bare devices were baked at $120^{\circ} \mathrm{C}$ for approximately $18 \mathrm{~min}$ to remove any residual organic solvent from the surfaces of the devices.

The $2 \%$ PIB solution was then spin cast at $2000 \mathrm{rpm}$ for $30 \mathrm{~s}$ onto the bare SAW device. The freshly coated devices were baked at $100^{\circ} \mathrm{C}$ for $\mathrm{l} \mathrm{hr}$ in a vacuum oven (pressure was approximately 1 Torr) to remove any residual chloroform. In addition to the SAW devices, a silicon coupon was also coated using the same procedure and the thickness was measured using a sloan Dektak profilometer. The thickness of the PIB films prepared as described was found to be approximately $1800 \AA$.

\subsection{TEMPERATURE STABILITY}

The freshly coated SAW devices were placed in the reference and signal SAW cases. In addition, two thermistors ( $5 \mathrm{k} \Omega$ at $25^{\circ} \mathrm{C}$ ) were placed in close thermal contact to the sAW cases to provide an independent measurement of the SAW case temperature. The unit was then closed, placed in a styrofoam insulating box, and allowed to warm to operating temperature.

Next, the room temperature was cycled between $14^{\circ} \mathrm{C}$ and $26^{\circ} \mathrm{C}$ with the sensing SAW device exposed to room air while the difference frequency was monitored via computer. Figure 4.1 
(zH) Kouanbad」 $\nabla$

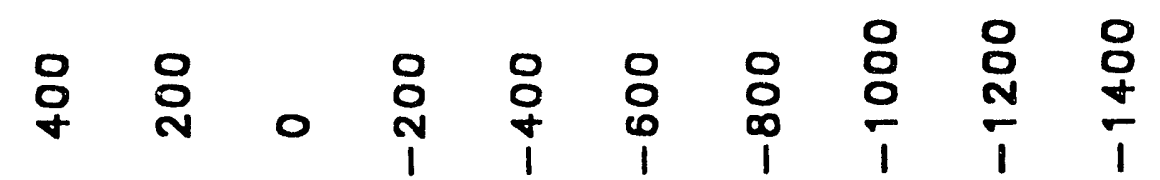

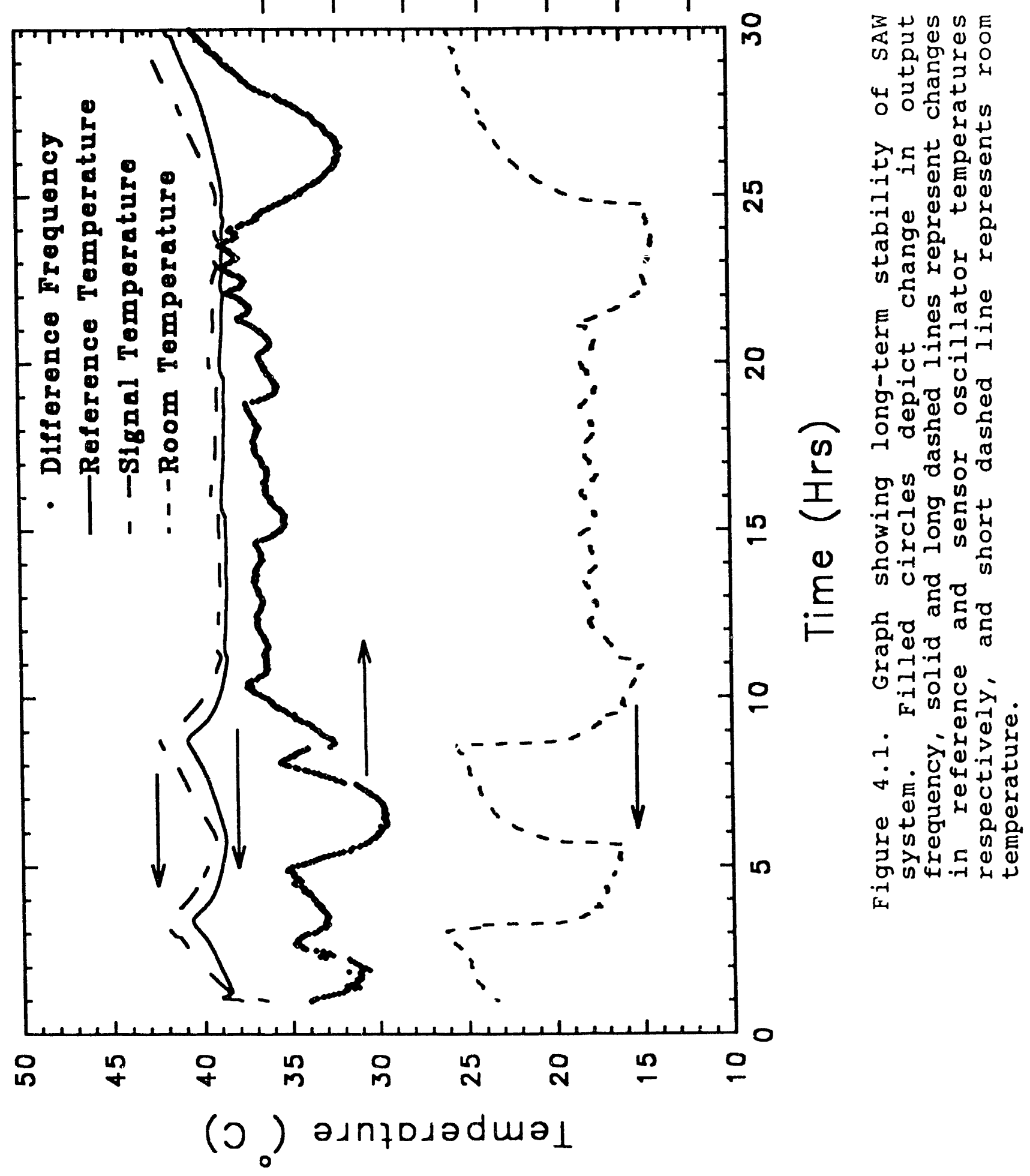


shows the difference frequency along with room, sensor SAW case, and reference SAW case temperatures plotted versus time. The room temperature was allowed to fluctuate rapidly over a span of $12^{\circ} \mathrm{C}$, resulting in a difference frequency shift of about $450 \mathrm{~Hz}$ over $30 \mathrm{hrs}$. Rapid shifts in room temperature $\left(>5^{\circ} \mathrm{C} / \mathrm{hr}\right)$ result in a larger change than do slower temperature shifts. Examination of the interval from 5 to $10 \mathrm{hrs}$ indicates that as the temperature of the room rose from $16^{\circ} \mathrm{C}$ to $26^{\circ} \mathrm{C}$, the frequency first dropped by $300 \mathrm{~Hz}$ and then rose by $300 \mathrm{~Hz}$. The sign change is a result of a thermal phase delay between the temperature of the signal (master) SAW case and the reference (slave) SAW case. Since the signal SAW case was bettex coupled to the environment, its temperature initially rose faster than the reference SAW case; however, as the reference sAw temperature rose to compensate for the change, the sign of the effect changed and the net temperature effect was eliminated. When the room temperature shift is slower, the phase delay becomes less significant, resulting in a smaller temperature difference between the two devices and a smaller difference-frequency shift. Ideally, to minimize the temperature drift further, both saW devices should be placed in a single case so that equal temperature changes are experienced. This approach is planned for future generations of this sensor.

During the interval in which the room temperature is most stable ( 11 to $21 \mathrm{hrs}$ ), the frequency fluctuates on the order of $150 \mathrm{~Hz}$. Thus, the minimum detectable signal over a 10 hour run 
with room temperature stability of $\pm 2^{\circ} \mathrm{C}$ is on the order of 200 $\mathrm{Hz}$.

A gas flow system (Figure 4.2) was constructed to calibrate the response of the SAW sensor to $\mathrm{CCl}_{4}$ exposure. Ultra high purity (99.999\%) $\mathrm{N}_{2}$ is used as a carrier gas and is passed through a gas washing bottle containing $\mathrm{CCl}_{4}$. The $\mathrm{CCl}_{4}$-saturated flow is mixed with another known flow of $\mathrm{N}_{2}$ to adjust the concentration and the resultant $\mathrm{CCl}_{4}$-laden stream is then passed by the SAW sensor and out the vent. The $\mathrm{CCl}_{4}$-laden stream diffuses from the main tube to the sensor and the difference frequency from the SAW device is monitored as a function of time. The steady-state frequency shift was recorded vs. $\mathrm{CCl}_{4}$ concentration.

Figure 4.3 is a plot of the change in frequency vs. $\mathrm{CCl}_{4}$ concentration in dry $\mathrm{N}_{2}$. The measured data (points) indicate that the sensor responds linearly to concentration with a slope of $1.1 \mathrm{ppm} / \mathrm{Hz}$. Sensor response is linear in the infinite dilution limit (Raoult's Law) but saturation is expected at much higher concentrations. In the application to be described, the concentrations are low and the system is assumed to be linear. All concentration vs. frequency change data are presented with frequency as the independent variable in order to simplify conversion of measured frequency shifts into concentrations during later experiments.

The above discussion of the frequency noise limit leads to an estimate for the minimum $\mathrm{CCl}_{4}$ detection limit of $200 \mathrm{ppm}$. The 


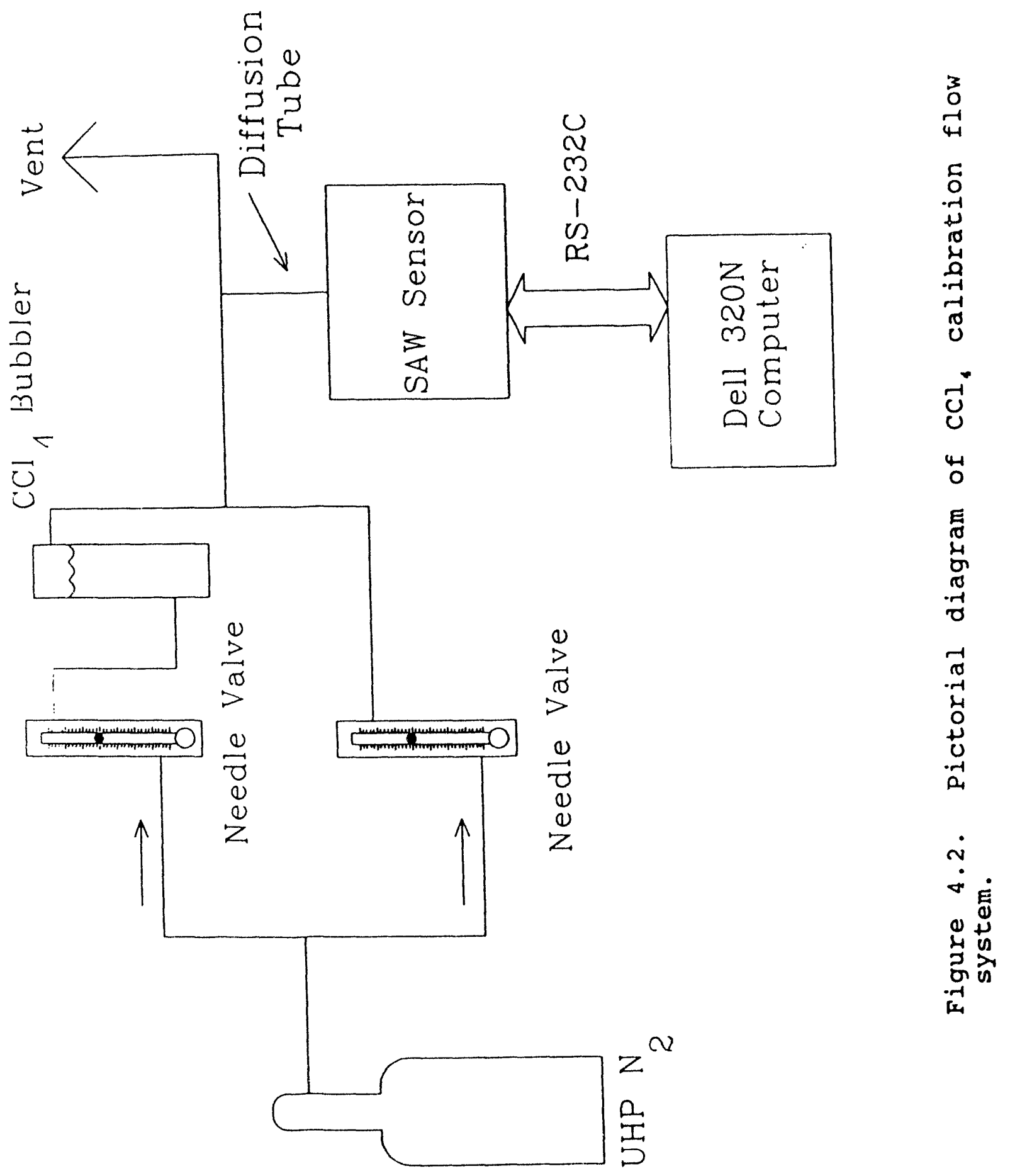




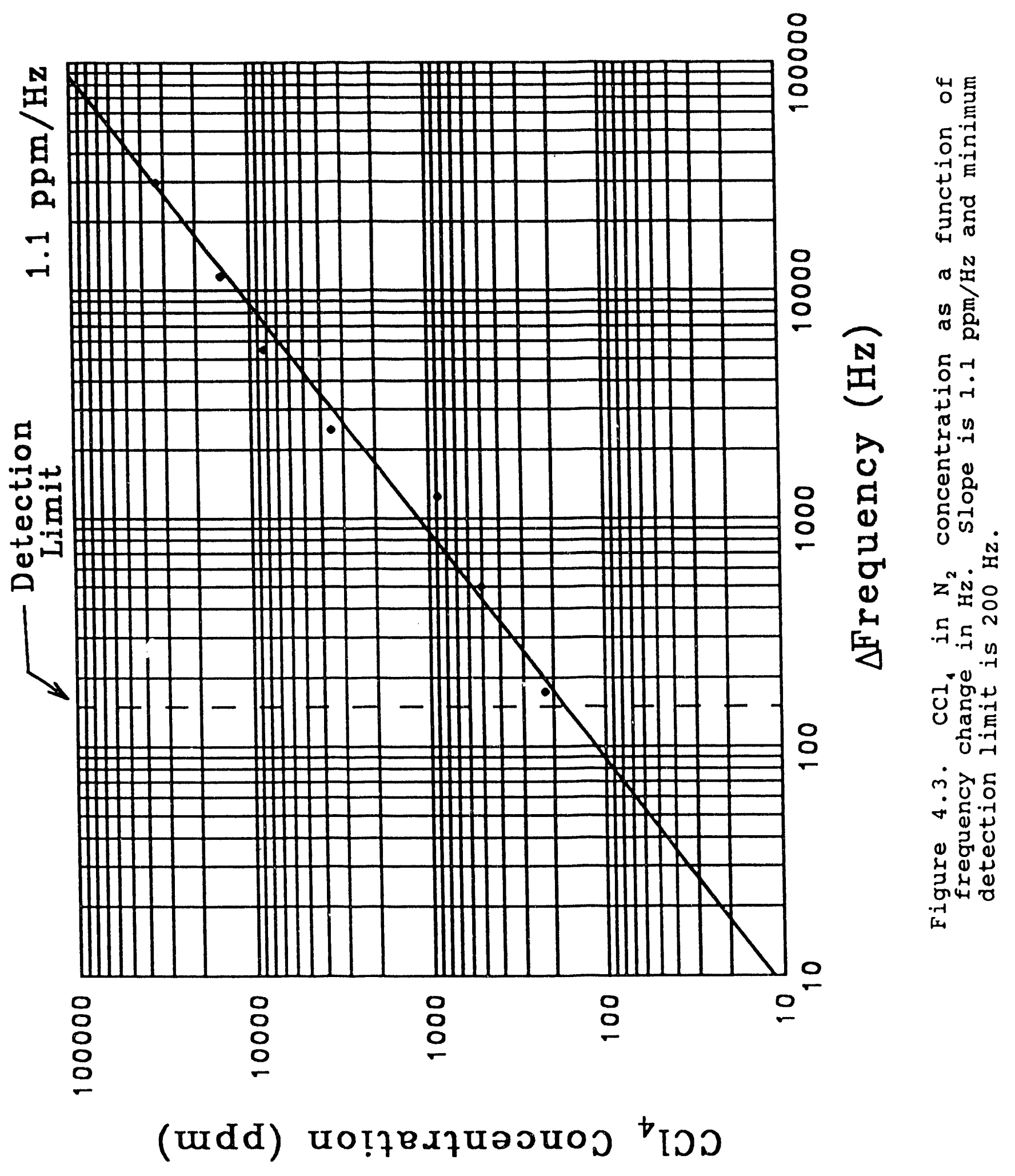


minimum detection limit can be lowered substantially by modifying the gas sampling technique so that the SAW sensor can be exposed quickly to a reference atmosphere and then exposed to the sample gas of interest at a rate much faster then the temperature drift. This could be accomplished by evacuation of the signal SAW sensor volume, monitoring the response of the saw to the vacuum, back-filling the volume with gas from the atmosphere of interest, and then observing the frequency change between the reference atmosphere and the sample atmosphere.

In the previous sections, a discussion of the laboratory tests of the sensor was presented. This included a description of the method for coating PIB films onto the SAW devices. In addition, thermal stability was discussed. It was found that the system has a larger temperature dependence for rapidly changing environmental temperatures. In the next generation of system development, the signal and reference device temperatures will be more closely thermally coupled by placing both the sensor and reference SAW devices in the same metal SAW case. Finally, the concentration dependence of the response was measured and found to be linear with a slope of $1.1 \mathrm{ppm} / \mathrm{Hz}$, indicating a minimum detection limit of about $200 \mathrm{ppm} \mathrm{CCl}_{4}$ in $\mathrm{N}_{2}$. In the next section, the field test results of the device are presented.

\subsection{FIELD TESTS}

This sensor was designed specifically to monitor the level of $\mathrm{CCl}_{4}$ that is emitted from the synthetica moving bed evaporator 
(MBEv). To accomplish this, several field trials of the system were performed at synthetica's Richmond, CA facility. In the following section, the results of the these trials are presented. First, the response as a function of $\mathrm{CCl} 4$ concentration in $\mathrm{CO}_{2}$ and steam was measured to determine the effects of a moist, corrosive environment on the function of the SAW sensor. Next, small-scale steam reforming of $\mathrm{CCl}_{4}$ in the synthetica quartz reactor was monitored and the sensor response was measured. Finally, large-scale steam reforming of $\mathrm{CCl}_{4}$ in the MBEv was demonstrated.

\subsection{QUARTZ REACTOR}

During the period of March 23-25, 1992, the sensor was tested on the synthetica quartz reactor depicted in Figure 5.1. The reactor consists of a steam source with a controllable feed rate and $\mathrm{a} \mathrm{CO}_{2}$ source connected through a $\mathrm{CCl}_{4}$ gas washing bottle. The $\mathrm{CCl}_{4}$-saturated stream was mixed with steam and piped into a furnace where the $\mathrm{CCl}_{4}$ was reformed into $\mathrm{CO}, \mathrm{CO}_{2}, \mathrm{H}_{2} \mathrm{O}$, and $\mathrm{HCl}$. The exhaust stream was then passed by the inlet to the SAW sensor and the $\mathrm{CCl}_{4}$ level measured with the output of the sensor recorded as a function of time by the notebook computer.

The first experiment on the quartz reactor was designed to determine the $\mathrm{CCl}_{4}$ concentration dependence of the SAW sensor response in $\mathrm{CO}_{2}$ and steam and what effect a corrosive steam atmosphere would have on the sensor. The data as a function of time are presented in Figure 5.2 as follows: Interval 1 is the 


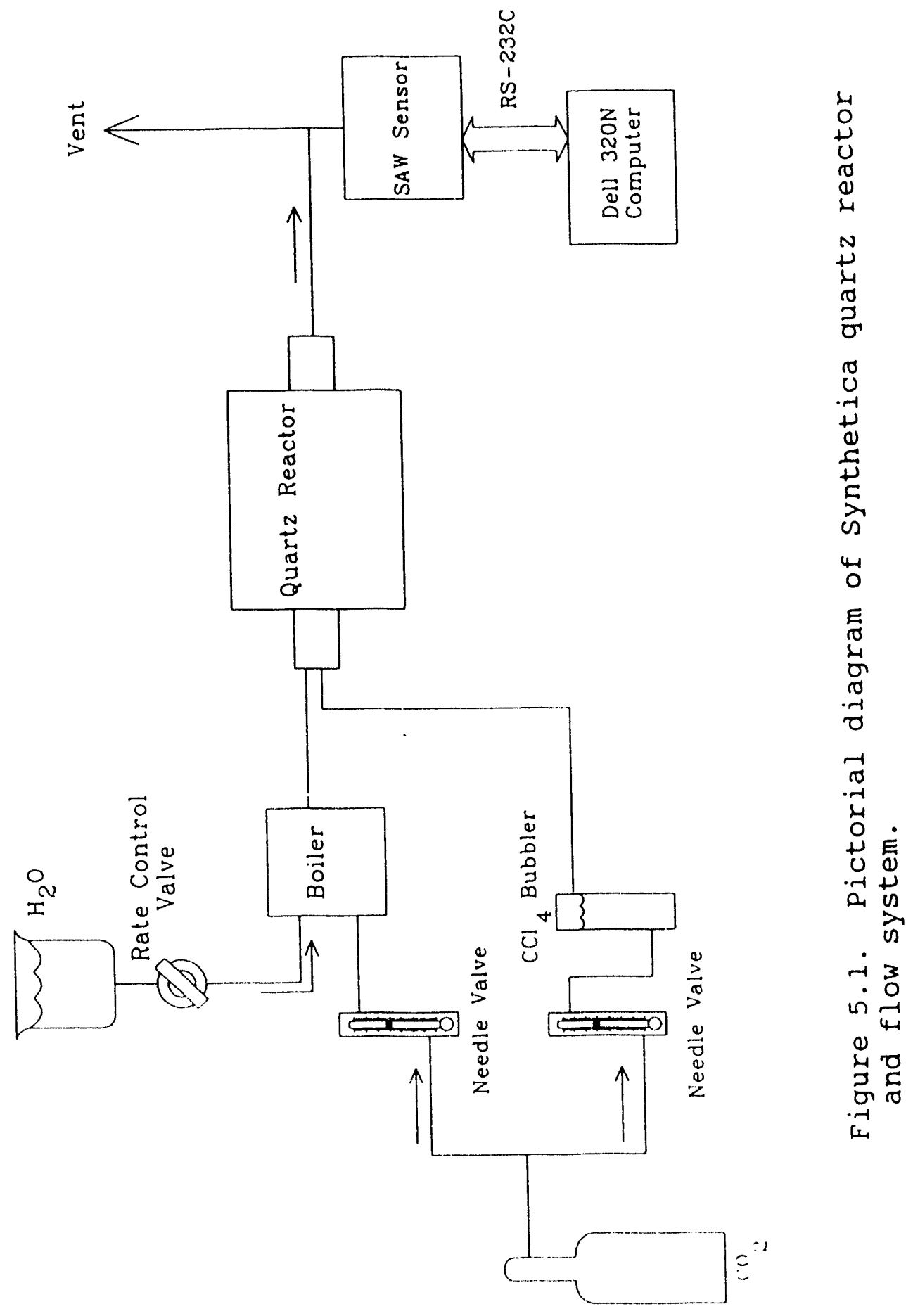


response for $12900 \mathrm{ppm} \mathrm{CCl}$, in dry $\mathrm{CO}_{2}$. Intervals 2 through 5 are various concentrations of $\mathrm{CCl}_{4}$ in steam with the various flow rates and $\mathrm{CCl}_{4}$ vapor pressures for each peak given in Table 5.1 . The variation in the $\mathrm{CCl}$, vapor pressure is a result of temperature changes in the room and radiative heating of the $\mathrm{CCl}_{4}$ reservoir by the biler heaters.

Table 5.1. Flow data used to calculate $\mathrm{CCl}$, concentrations in synthetica quartz reactor.

\begin{tabular}{|c|c|c|c|c|c|c|}
\hline $\begin{array}{l}\text { INTER. } \\
(\#)\end{array}$ & $\begin{array}{c}\mathrm{CCl} \\
\text { FLo' } \\
\text { (mL/min) }\end{array}$ & $\begin{array}{c}\mathrm{CO}_{2} \\
\text { FLOW } \\
(\mathrm{mL} / \mathrm{min})\end{array}$ & $\begin{array}{l}\mathrm{H}_{2} \circ(g) \\
\text { FLOW } \\
(\mathrm{mL} / \mathrm{min})\end{array}$ & $\begin{array}{l}\mathrm{CCl}_{4} \\
\text { PRESS. } \\
\text { (Torr) }\end{array}$ & $\begin{array}{l}\mathrm{CCl}_{4} \\
\text { CONC. } \\
\text { (ppm) }\end{array}$ & $\begin{array}{l}\Delta \nu \\
(\mathrm{kHz})\end{array}$ \\
\hline $\begin{array}{l}1 \\
2 \\
3 \\
4 \\
5\end{array}$ & $\begin{array}{l}523 \\
522 \\
265 \\
265 \\
532\end{array}$ & $\begin{array}{l}3161 \\
3161 \\
3161 \\
6322 \\
1611\end{array}$ & $\begin{array}{l}0 \\
1060 \\
1350 \\
690 \\
1270\end{array}$ & $\begin{array}{l}69.8 \\
80.3 \\
80.3 \\
80.3 \\
80.3\end{array}$ & $\begin{array}{r}12900 \\
11500 \\
5830 \\
3830 \\
16200\end{array}$ & $\begin{array}{l}15.9 \\
16.2 \\
12.3 \\
7.3 \\
25.4\end{array}$ \\
\hline
\end{tabular}

a. Atmospheric pressure was 760 Torr. Quartz reactor temperature was $330^{\circ} \mathrm{C}$.

b. Concentration in ppm by volume.

The concentrations in Table 5.1 are calculated based on an atmospheric pressure of 760 Torr (Richmond, CA has an elevation of approximately $20^{\prime}$ above sea level). Initially, the quartz reactor was kept at a temperature of $330^{\circ} \mathrm{C}$ to avoid destroying the $\mathrm{CCl}_{4}$ and the steam boiler was kept at an approximately constant temperature of $550^{\circ} \mathrm{C}$.

The arrows in Figure 5.2 indicate when the sensor test case volume was evacuated using a small diaphragm vacuum pump "nd a three way valve to switch between vacuum and sample stream. As indicated, the response diopped to baseline rapidly and then returned to a signal level when the volume was backfilled with a 


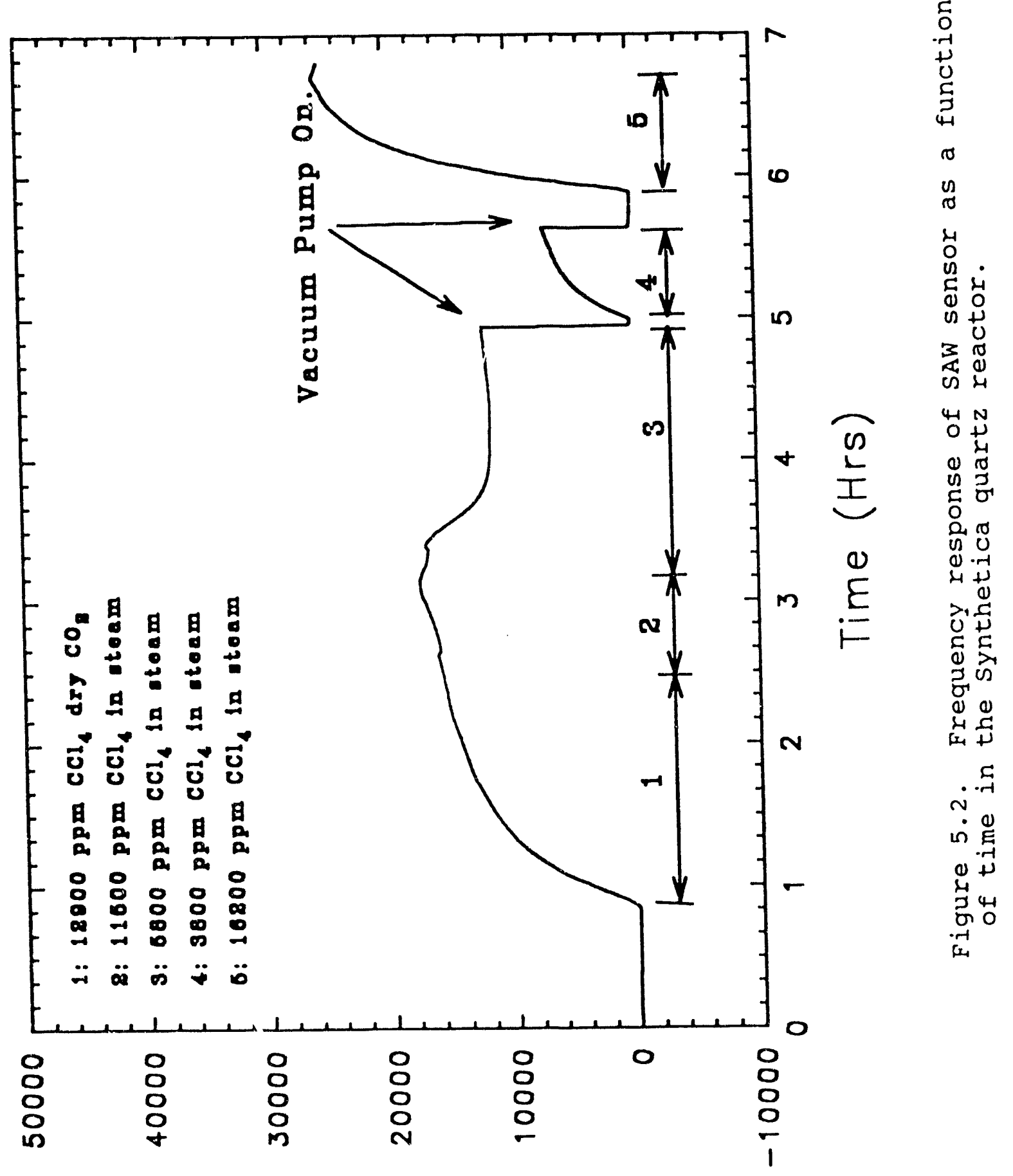

$$
\text { (zH) Kouanbaj }
$$


$\mathrm{CCl}_{4}$-laden stream. The responses in Intervals 4 and 5 on the plot are not significantly faster for the backfilled sampling technique over the diffusion sampling technique of Interval 1 . This is due to a leak in the system that brought the sample chamber to atmospheric pressure before the valve could be switched. However, further testing in the laboratory with the backfill sampling technique indicates that an order of magnitude increase in response time can be achieved by the backfill technique over the diffusion technique.

Figure 5.3 is a plot of the change in frequency versus $\mathrm{CCl}_{4}$ concentration for the concentrations listed in Table 5.1. The responses measured in the presence of steam are plotted as filled circles and the data point measured in ary $\mathrm{CO}_{2}$ is plotted as an upside-down triangle. Error bars are calculated assuming an uncertainty in the $\mathrm{CCl}_{4}$ flow rate of $10 \mathrm{~mL} / \mathrm{min}$, an uncertainty in the steam and $\mathrm{CO}_{2}$ flow rates of $100 \mathrm{~mL} / \mathrm{min}$, and an uncertainty in the temperature of the $\mathrm{CCl}_{4}$ of $1^{\circ} \mathrm{C}$. Figure 5.3 shows that, within these uncertainties, the sensor responded linearly and has a slope of $0.6 \mathrm{ppm} / \mathrm{Hz}$.

Next, with a fixed $\mathrm{CCl}_{4}$ concentration of $16200 \mathrm{ppm}$ in steam (mixture 5 in Table 5.1), the temperature of the quartz reactor was slowly ramped from $330^{\circ} \mathrm{C}$ to $560^{\circ} \mathrm{C}$. The change in SAW frequency and corresponding change in $\mathrm{CCl}_{4}$ concentration are plotted as a function of temperature in Figure 5.4 with circles representing the data from the SAW sensor. The solid line is the $\mathrm{CCl}$, concentration as a function of temperature for pyrolysis as 


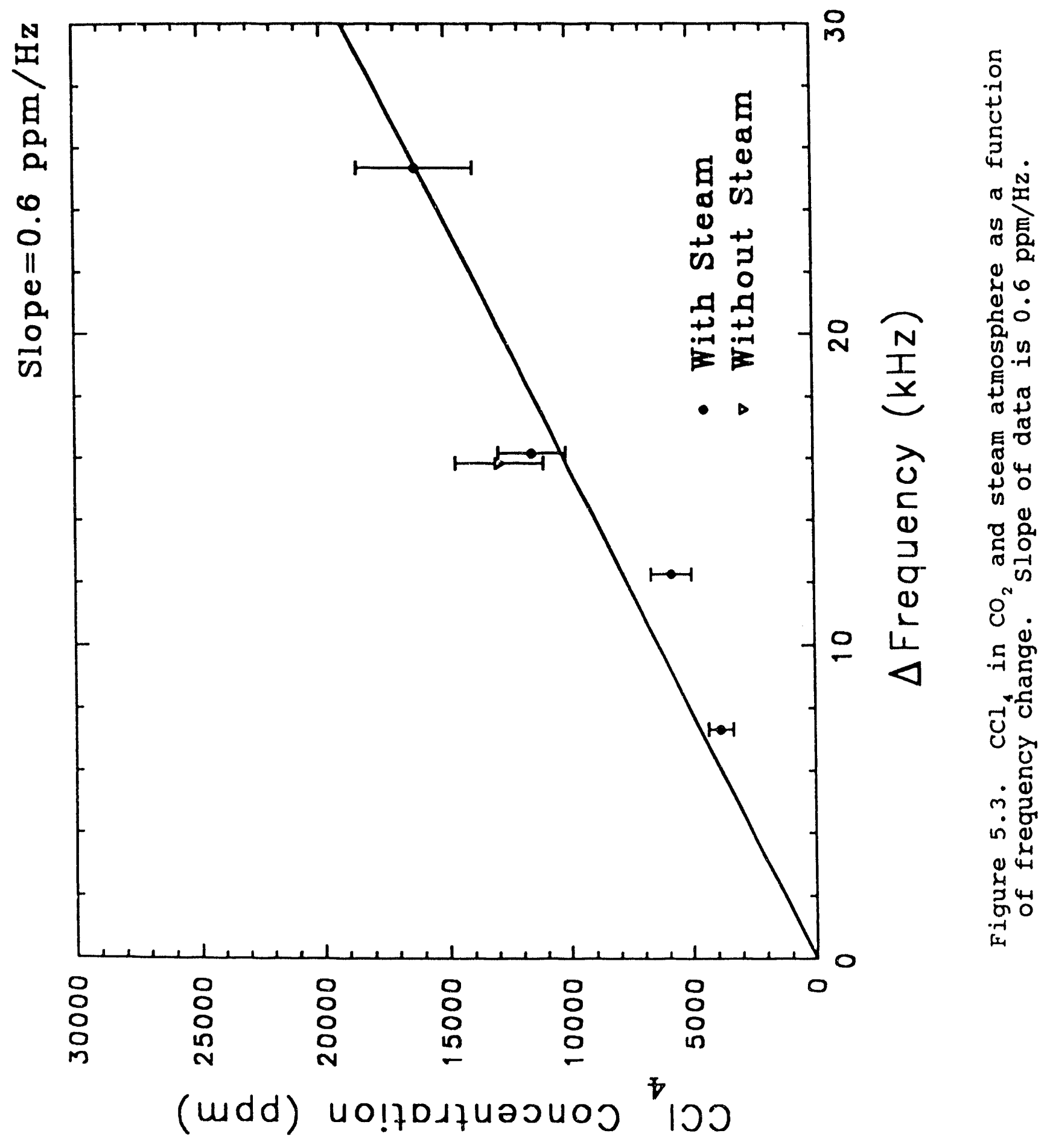




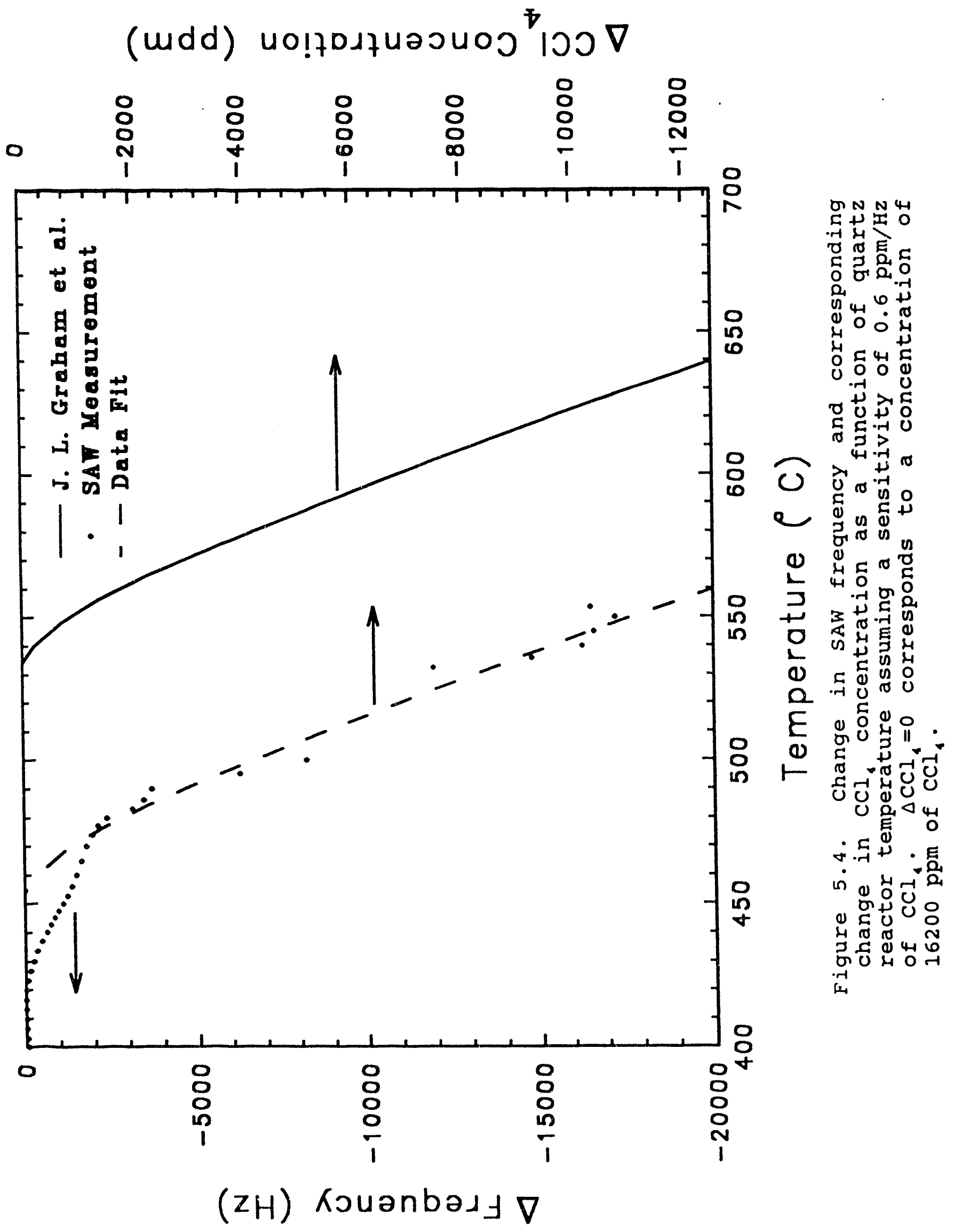


reported in the literature, and the dashed line is the literature data translated to the left by $80^{\circ} \mathrm{C} .21$ The literature data is reported as the weight percent remaining after the pyrolysis process at each temperature; this data was converted to a destruction efficiency and multiplied by our starting concentration to obtain a comparison.

It is clear that the steam reforming process is more efficient than pyrolysis since equal destruction occurs at a temperature that is $80^{\circ} \mathrm{C}$ lower in the steam reformer than in the pyrolysis chamber. This is due to the addition of steam and the greater resultant thermodynamic driving force associated with the formation of the oxidized products. Examination of the chemical equation that describes the $\mathrm{CCl}_{4}$ destruction process indicates that addition of $\mathrm{H}_{2} \mathrm{O}$ to the system results in more destruction of CCl, as Lechatelier's principle says it must.

$2 \mathrm{H}_{2} \mathrm{O}(\mathrm{g})+\mathrm{CCl}_{4}(\mathrm{~g})=\mathrm{CO}_{2}(\mathrm{~g})+4 \mathrm{HCl}(\mathrm{g}) \quad 5.1$

Additional destruction efficiency may in part be due to the longer residence time of the molecules in the reactor. The pyrolysis literature reports a residence time of at most $6 \mathrm{sec}$ while the synthetica quartz reactor residence time is on the order of $18 \mathrm{sec}$ with the flows given.

The next large scale test of the sensor occurred during oct. 13-15, 1992 and was designed to determine the destruction efficiency of the synthetica moving-bed evaporator (MBEv). A schematic diagram of the system is given in Figure 5.5 and shows 


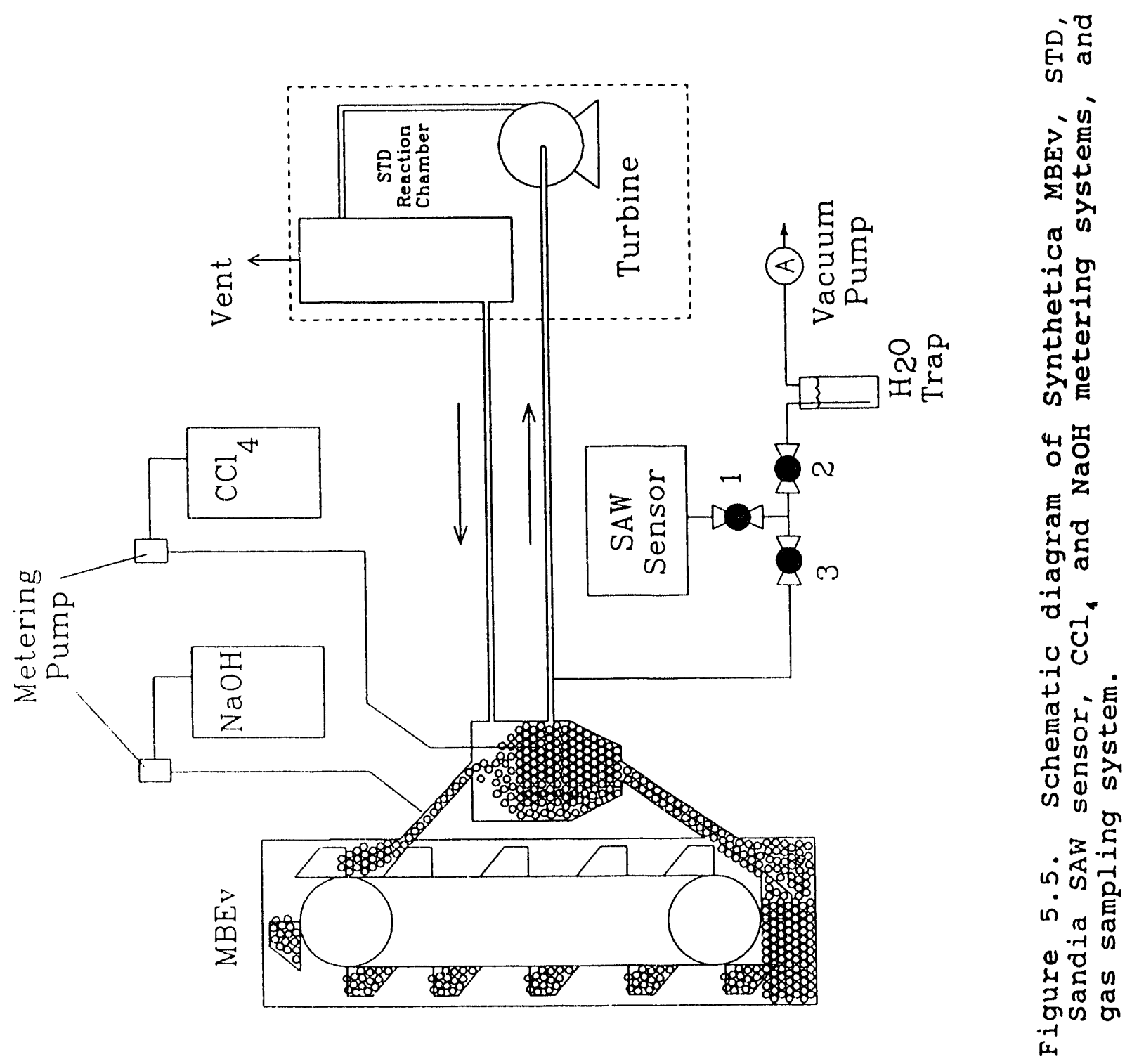


the MBEv, STD (Synthetica Technologies Detoxifier), SAW sensor, $\mathrm{CCl}_{4}$ delivery system, $\mathrm{NaOH}$ delivery system, and gas sampling mechanism. The hazardous waste (in this case $\mathrm{CCl}_{4}$ ) is introduced directly into the $\mathrm{MBEv}$ where it is steam reformed into $\mathrm{CO}, \mathrm{CO}_{2}$, hydrocarbon fragments, and mineral acids such as $\mathrm{HCl}$.

As depicted in Figure 5.5, the MBEv consists of a bucket elevator system to lift vitreous $\mathrm{Al}_{2} \mathrm{O}_{3}$ spheres (3/8" diameter) to the top of a hopper where they are coated with $\mathrm{NaOH}$ and allowed to settle to the bottom of the hopper. In the entry arm of the hopper, the moving spheres roll through a stream of concentrated $\mathrm{NaOH}$ that coats their surfaces. Neutralization of any molecules of mineral acid produced by steam reforming in the MBEv occurs by contact with the $\mathrm{NaOH}$ coated spheres. The $\mathrm{Al}_{2} \mathrm{O}_{3}$ bed consists of about 600 lbs of $3 / 8 "$ spheres and represents about $50 \mathrm{~m}^{2}$ of surface area.

The output gas from the MBEv is pumped by the turbine into the higher temperature $\left(1100^{\circ} \mathrm{C}\right)$ reaction chamber of the STD where any remaining organic fragments are converted to $\mathrm{CO}, \mathrm{CO}_{2}$ and $\mathrm{H}_{2} \mathrm{O}$ and then vented to the atmosphere. It is absolutely necessary that the total $C l$ concentration be kept below two weight percent or serious damage will occur to the STD vessel and heating elements. Thus, the SAW sensor was placed on the outlet of the MBEv to monitor the output $\mathrm{CCl}$, level from the MBEv and hence determine its efficiency at destroying the $\mathrm{CCl}_{4}$ and protecting the STD. With the addition of a $\mathrm{pH}$ monitoring apparatus, it is possible to assess the efficiency of the MBEv at 
removing the $\mathrm{CCl}_{4}$ from the system and determine the level of acid neutralization provided by the NaOH-coated alumina spheres.

The experimental procedure employed was as follows: first, with the MBEv effluent steam stream at $230^{\circ} \mathrm{C}$ (a temperature too low to cause $\mathrm{CCl}_{4}$ to be destroyed by the steam reforming), $\mathrm{CCl}_{4}$ was introduced into the system via the metering apparatus while the SAW sensor monitored a sample of gas from the MBEv outlet. The flow rate was kept below $10 \mathrm{ml} / \mathrm{min}$ to keep the total $\mathrm{cl}$ concentration below 2\%. The gas was sampled by pumping a fresh volume of sample gas from the MBEv outlet with valves 2 and 3 open and Valve 1 closed. Next, Valve 3 was closed so that the sensor could be evacuated, and the sensor was backfilled with the sample gas by first closing Valve 2 and then opening Valves 3 and 1 in order. A calibration point was measured that indicated a shift of $800 \mathrm{~Hz}$ for a calculated concentration of $1228 \mathrm{ppm}$. From the quartz reactor experiments, it was shown that the frequency response is a linear function of concentration; thus, a scale factor of $1.5 \mathrm{ppm} / \mathrm{Hz}$ was found from this calibration measurement. The calibration found for the quartz reactor measurement $(0.6$ ppm/Hz) was not in agreement with this value and was ignored. This inconsistency was probably due to uncertainties in the steam flow rate in the synthetica equipment, uncertainties in the $\mathrm{CCl}_{4}$ flow rate, and errors introduced by condensation of $\mathrm{CCl}_{4}$ on the walls of the diffusion tube.

Next, the concentration was changed and the above process was repeated as the temperature of the MBEv steam stream was 
increased causing steam destruction of $\mathrm{CCl}_{4}$ by steam reforming. These data are tabulated in Table 5.2 .

Table 5.2. Data collected from MBEv and SAW sensor as a function of MBEv outlet temperature.

\begin{tabular}{||c|c|c|c|c|c||}
\hline $\begin{array}{c}\text { NaOH } \\
\text { flow } \\
(\mathrm{ml} / \mathrm{min})\end{array}$ & $\begin{array}{c}\mathrm{CCl} \\
\text { flow } \\
(\mathrm{ml} / \mathrm{min})\end{array}$ & $\begin{array}{c}\text { CCl } \\
\text { Measured } \\
(\mathrm{ppm}) \mathrm{a}\end{array}$ & $\begin{array}{c}\text { CCl } \\
\text { Calculated } \\
(\mathrm{ppm})\end{array}$ & $\begin{array}{c}\text { MBEv Temp. } \\
\text { inlet/outlet } \\
\left({ }^{\circ} \mathrm{C}\right)\end{array}$ & $\begin{array}{c}\xi \\
(\%)\end{array}$ \\
\hline 0 & 6.2 & 1200 & 1230 & $497 / 236$ & 98 \\
0 & 4.6 & 975 & 924 & $516 / 243$ & 106 \\
0 & 3.0 & 300 & 600 & $517 / 272$ & 50 \\
0 & 7.0 & 750 & 1390 & $517 / 272$ & 54 \\
50 & 8.0 & 460 & 1590 & $522 / 297$ & 29 \\
60 & 10 & 300 & 1990 & $501 / 283$ & 7 \\
60 & 20 & 280 & 3980 & $503 / 281$ & 8 \\
120 & 20 & 300 & 3980 & & \\
\hline
\end{tabular}

a. At outlet from MBEv.

b. At inlet to MBEv.

c. Percent of inlet $\mathrm{CCl}_{4}$ remaining at outlet of MBEv.

In Table 5.2, the measured $\mathrm{CCl}_{4}$ level at the MBEv outlet is the product of the measured frequency change in $\mathrm{Hz}$ and the scale factor 1.5 discussed earlier. The calculated $\mathrm{CCl}_{4}$ concentration at the MBEv inlet is derived as follows:

$$
\mathrm{ppm} \mathrm{CCl}_{4}=\frac{\mathrm{R} \rho}{\mathrm{MW} \beta \wp} \times 10^{\mathrm{B}}
$$

where $\rho$ is the density of $\operatorname{ccl}_{4}(1.6 \mathrm{~g} / \mathrm{ml})$, MW is the molecular weight of $\operatorname{CCl}_{4}(154 \mathrm{~g} / \mathrm{mole}), \beta$ is the measured steam flow rate in the machine $(45 \mathrm{scfm}), R$ is the $\mathrm{CCl}_{4}$ flow rate into the machine, and $p$ is the conversion ratio from 
scfm to moles steam ( 1.16 moles/scfm).

The parameter $\xi$ in Table 5.2 is the ratio of the measured $\mathrm{CCl}_{4}$ outlet level to the calculated $\mathrm{CCl}_{4}$ inlet level converted to percent. This parameter is plotted as a function of MBEv outlet temperature in Figure 5.6 and shows a large decrease with increasing temperature. This decrease indicates that $\mathrm{CCl}_{4}$ is being steam reformed into its reaction products, and that the MBEv is capable of destroying at least $90 \%$ of the input $\mathrm{CCl}_{4}$ at MBEv inlet and outlet temperatures of $500^{\circ} \mathrm{C}$ and $300^{\circ} \mathrm{C}$ respectively. As indicated in the table, the inlet temperature of the MBEv is much higher than the outlet temperature, indicating that the $\mathrm{CCl}_{4}$ is actually exposed to a bed whose temperature is somewhere between the temperature of the inlet and the outlet. Measurement of the actual moving-bed temperature is difficult due to the constant motion of the spheres; thus, the destruction temperature of $\mathrm{CCl}_{4}$ in the MBEv can only be inferred from the inlet and outlet temperatures.

A significant limitation of the destruction capacity of the MBEv is the heating capacity of the steam from the STD. The MBEV has no steam generation or heating capacity of its own and therefore is heated by steam passing from the STD through the turbines to the MBEv. As indicated in Table 5.2, the temperature of both the inlet and outlet of the MBEv began to drop with increasing $\mathrm{NaOH}$ and $\mathrm{CCl}_{4}$ flow. This was due to the cooling caused by the water in the $\mathrm{NaOH}$ solution evaporating as it came in contact with the hot spheres. While $\mathrm{NaOH}\left(\mathrm{MP}=318^{\circ} \mathrm{C}\right)$ is molten 


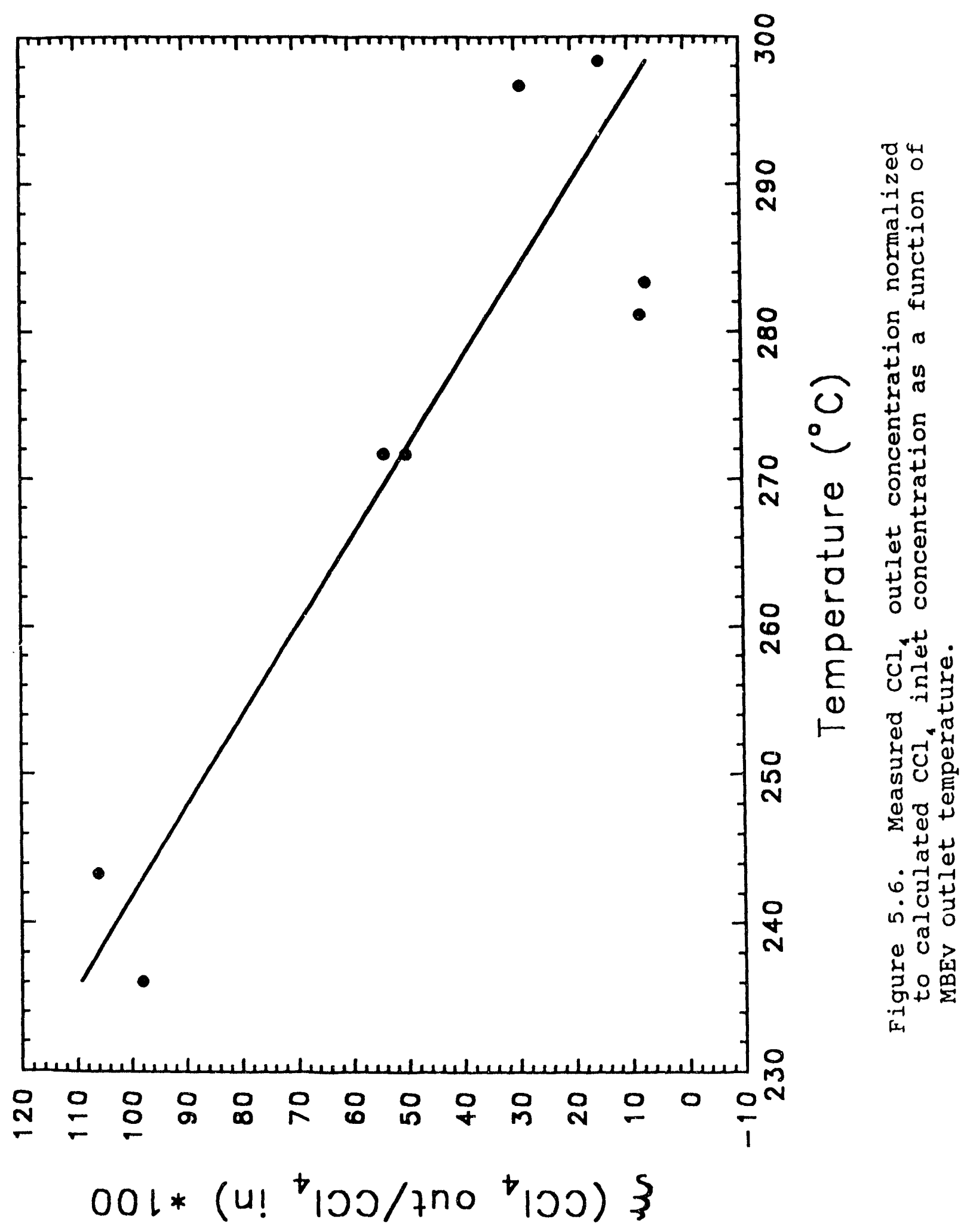


in the MBEv, too much cooling of the bed will cause the NaOH to freeze and convert the moving bed into a mass of solid $\mathrm{NaOH}$ and $\mathrm{Al}_{2} \mathrm{O}_{3}$ spheres that will jam the machine. To avoid this problem, the experiment was terminated before the MBEv cooled below the melting point of $\mathrm{NaOH}$. This premature termination of the experiment prevented testing with higher flow rates of $\mathrm{CCl}_{4}$, that in turn precluded demonstration of higher than $90 \%$ destruction.

As indicated in Figure 5.5, the gas sampling tubing had a water trap to prevent moisture from reaching the vacuum pump and destroying it. By measuring the $\mathrm{pH}$ of the water in the trap it was possible to qualitatively verify than the MBEv was producing acid (i.e., reforming $\mathrm{CCl}_{4}$ ) and that the $\mathrm{NaOH}$ was neutralizing the acid. Since the trap was clear glass, it was possible to place wide range $\mathrm{pH}$ paper in the water and visually monitor the color change, and thus the pH change. At several intervals during the course of the experiment, the $\mathrm{H}_{2} \mathrm{O}$ in the trap and the $\mathrm{pH}$ paper were changed and fresh samples of steam were pulled through the trap. It was verified that by running either an excess of $\mathrm{CCl}_{4}$ or $\mathrm{NaOH}$ it was possible to change the $\mathrm{pH}$ of the trap water from about 3 with excess $\mathrm{CCl}_{4}$ to 8 with excess $\mathrm{NaOH}$ in the MBEv. This verifies qualitatively that the MBEv was destroying $\mathrm{CCl}_{4}$ and neutralizing the $\mathrm{HCl}$ released by the destruction, and that the SAW measurements at high temperatures are correct.

In summary, through various experiments with the SAW sensor, 
it was possible to determine the following information about the operation of the SAW sensor and the MBEv. First, from the quartz reactor experiment, the SAW sensor was shown to respond linearly with $\mathrm{CCl}_{4}$ concentration in moist, corrosive background atmospheres with no discernable damage to the sensor (the SAW devices used in this system were constructed from $20 \AA$ of $\mathrm{Cr}$ and $2000 \AA$ of Au lithographically patterned on a quartz substrate; conventional SAW devices are patterned in Al and would corrode rapidly in this environment). It was also shown that the sensor can monitor destruction of $\mathrm{CCl}_{4}$. Second, it was shown that the SAW sensor can measure $\mathrm{CCl}_{4}$ concentrations in the harsh environment of the full-scale synthetica system. Finally, it was demonstrated that the synthetica MBEv can destroy $\mathrm{CCl}_{4}$ with at. lest $90 \%$ efficiency at MBEv inlet and outlet temperatures of $500^{\circ} \mathrm{C}$ and $300^{\circ} \mathrm{C}$ respectively.

\subsection{CONCLUSIONS}

In the above discussions, we have described a sensor based on surface acoustic wave technology for the measurement of vocs in very harsh chemical and physical environments. A mathematical description of the physical phenomena occurring in the SAW device was given to describe the sensing mechanism. Next, a detailed description of the mechanical and electrical construction of the device was presented. This included descriptions of the efforts made to minimize temperature effects on the response of the sensor and calibration of the device as a function of 
concentration of $\mathrm{CCl}_{4}$. It was found that the sensor is stable to on the order of $200 \mathrm{~Hz}$ over $10 \mathrm{hr}$ periods and that the sensor responds linearly to $\mathrm{CCl}_{4}$ concentration. A minimum detection limit of about $200 \mathrm{ppm} \mathrm{CCl}$ was given for industrial environments even though the sensor can be operated with better stability ir the laboratory,

Sensor operation was then demonstrated in the harsh physical and chemical en ironment of the synthetica MBEv and it was verified thal the MBEv can destroy a . inimum of $90 \% \mathrm{CCl}_{4}$ at $M B E V$ inlet and outlet temperatures of $500^{\circ} \mathrm{C}$ and $300^{\circ} \mathrm{C}$ respectively. From the measurements in the quartz reactor, the sensor was shown to function in a corrosive environment of $\mathrm{HCl}$ and steam and that using a vacuum pump to evacuate the SAW case and then backfilling the system with the VOC laden gas was an efficient method of increasing the response time in the sensor.

Finally, several recommendations for improvements in the sensor were suggested. For example, embedding both SAW devices in the same metal case would provide better thermal coupling and reduce the temperature induced fluctuations in the sensor. In addition, adding a voltage follower between the output of the op amp and the current switching transistor in the sensor heater control circuit will reduce the temperature induced feedback that is translated back to the resistance bridge and result in better long term stability of the system. Another recommended modification to the system would be the addition of a electrically actuated three-way valve and a vacuum pump to allow 
automatic switching between the sample gas and baseline.

Future additions to this type of system include the development of SAW devices with built-in resistance temperature devices (RTDS) for on chip measurement of the SAW surface temperature and additional interface hardware and software to allow compensation for temperature induced fluctuations in SAW frequency. Finally, integration of the system with a built-in microprocessor would allow stand alone operation and remote sensing via modem and telephone lines.

Future development of high temperature films would allow the direct sensing of gases within hot environments and reduce the problems associated with sensing via diffusion tubes. Currently there are no films that show the required sensitivity at temperatures much higher than the $40^{\circ} \mathrm{C}$ used here. It is clear that optimization of this type of system will require much further research and development; however, as we have shown, this type of system displays great potential for becoming the standard technology for measurements in harsh environments.

The authors would like to acknowledge R. W. Cernosek, L. Casaus, G. C. Frye, and J. L. Sprung of Sandia National Laboratories for their technical assistance and useful insights. In addition, we would like to thank Dr. T. R. Galloway and his staff at synthetica technologies for their technical assistance. This work was funded by the United States Department of Energy as part of the VOC Non-Arid and VOC Arid integrated demonstrations (Technical Task Plan (TTP) AL-221101 and AL-221106) and is 
supported by the US DOE under contract DE-AC04-76DP00789. 
68

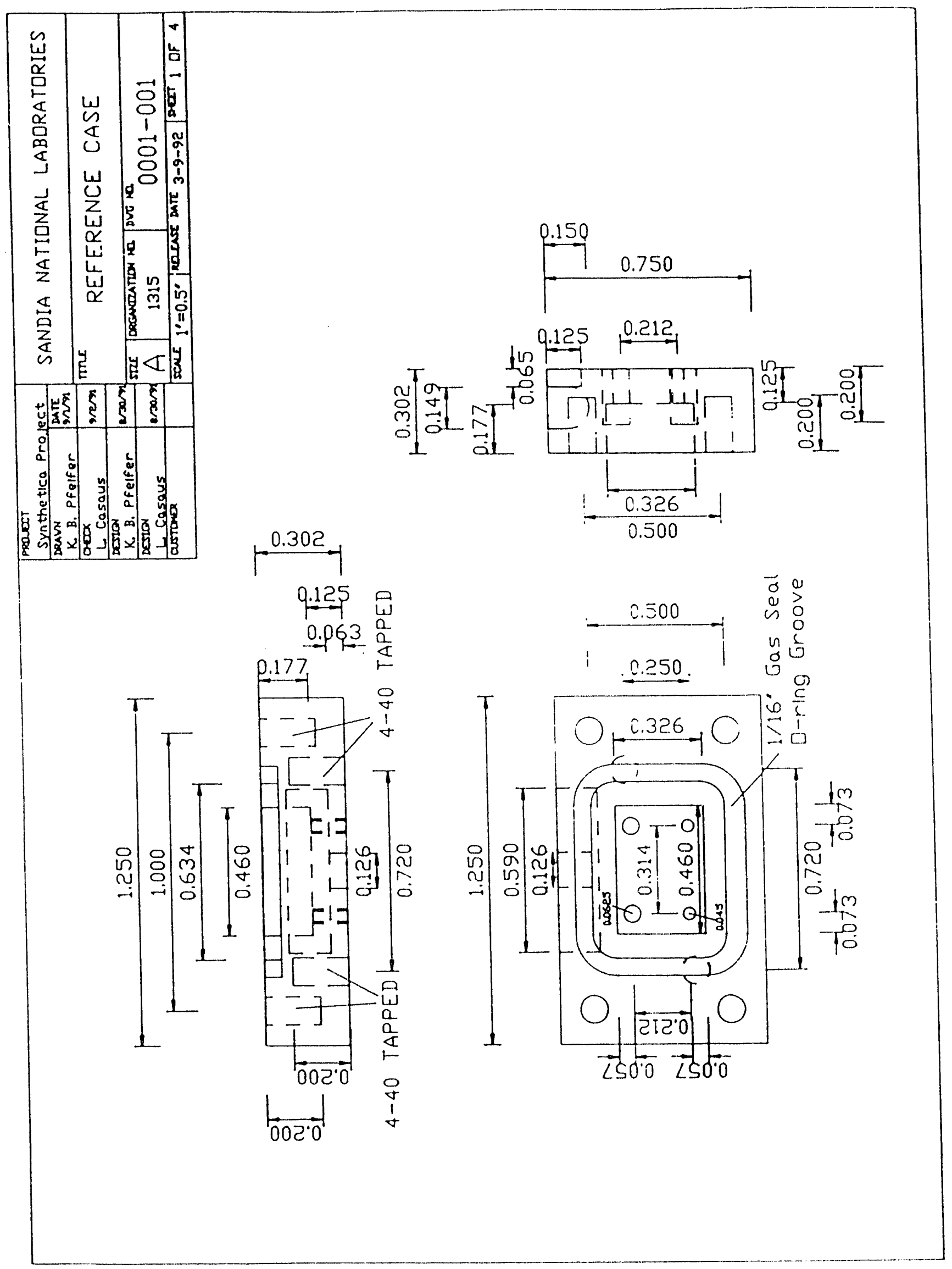

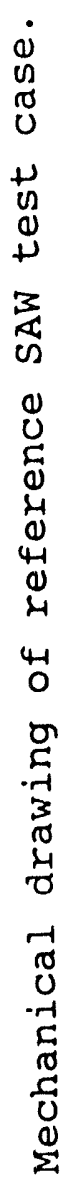

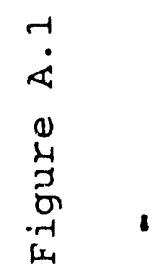




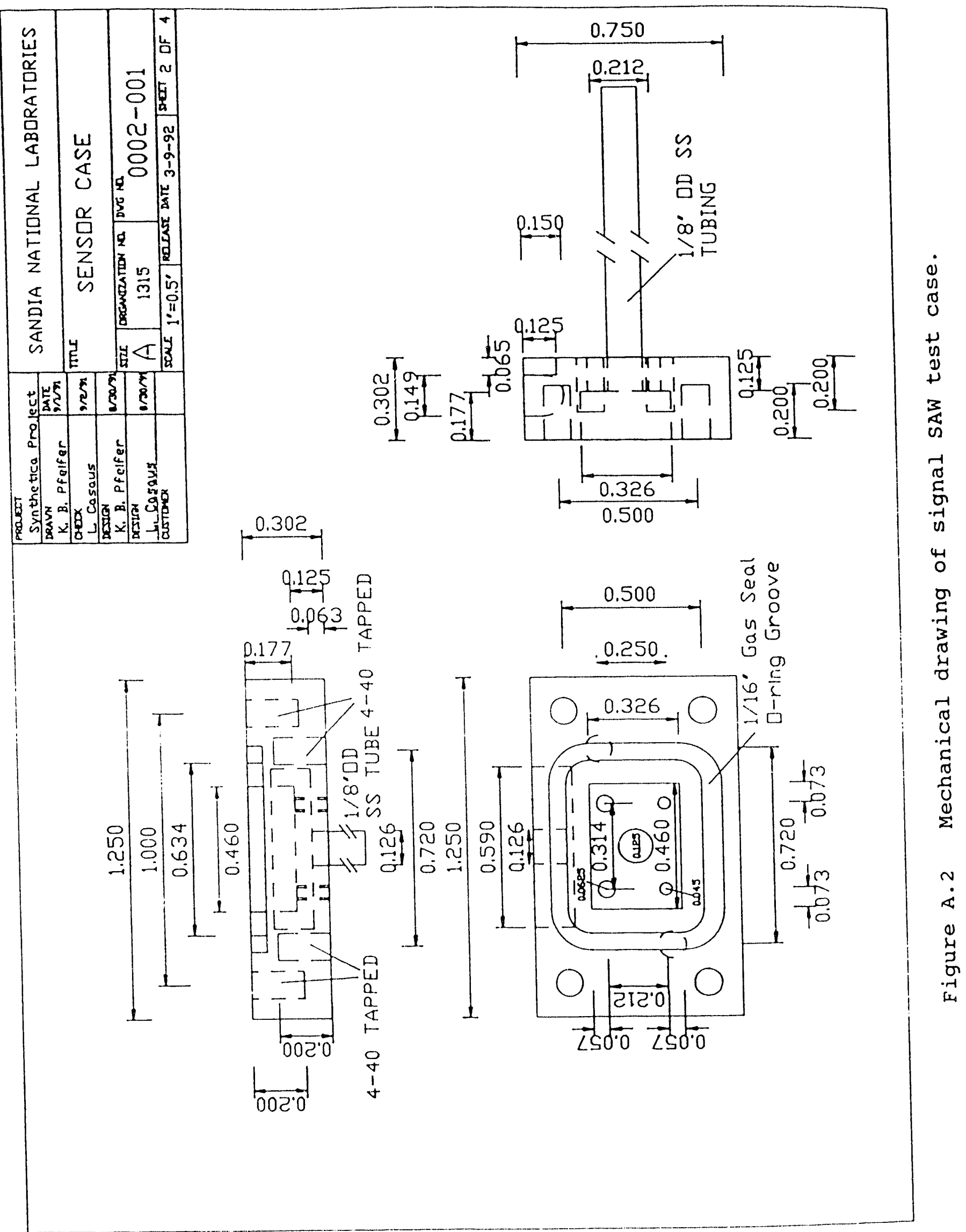




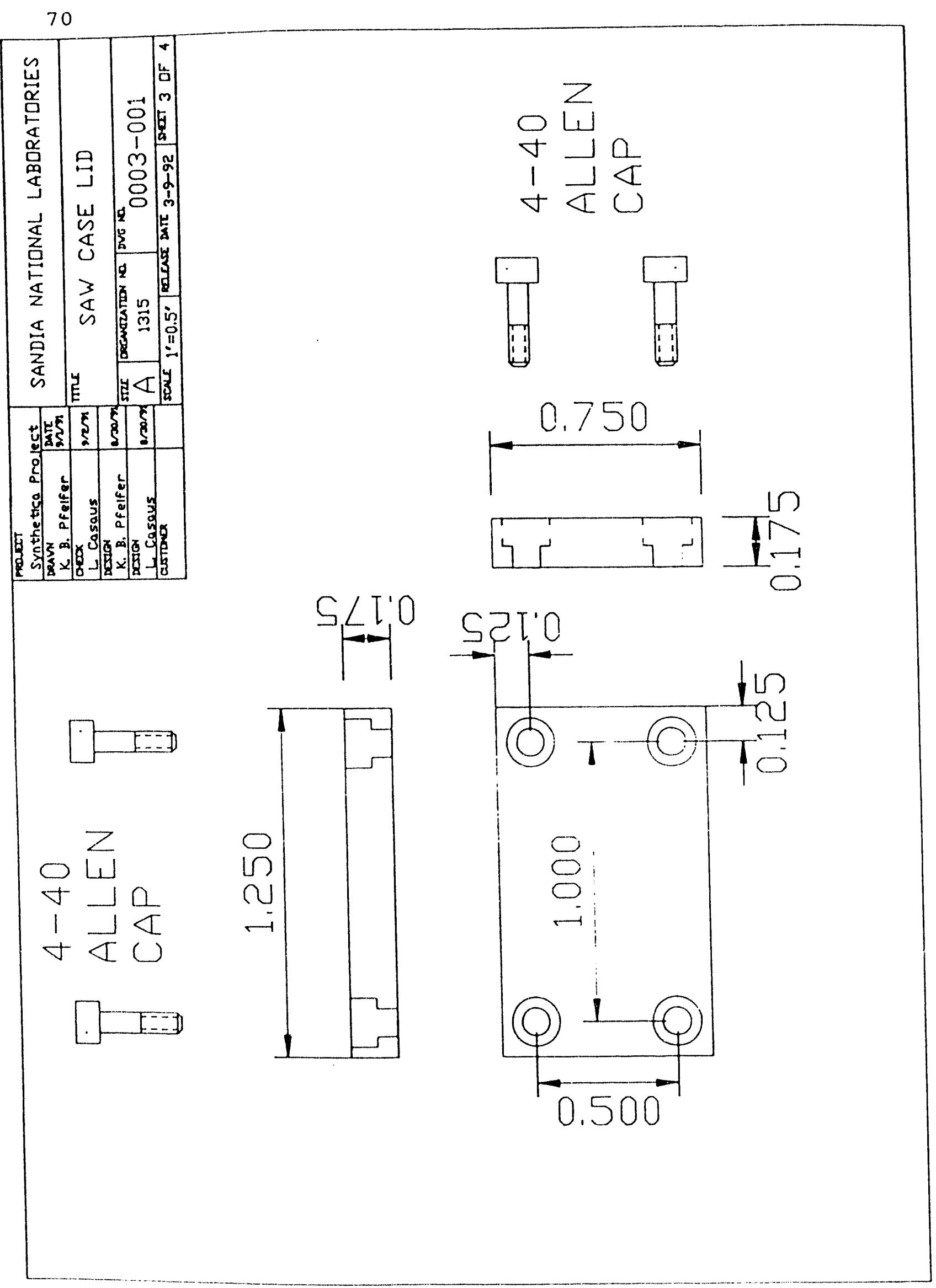

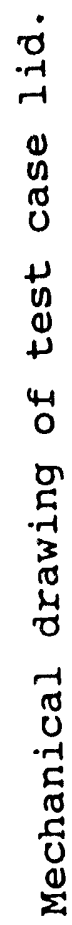

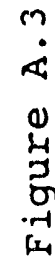




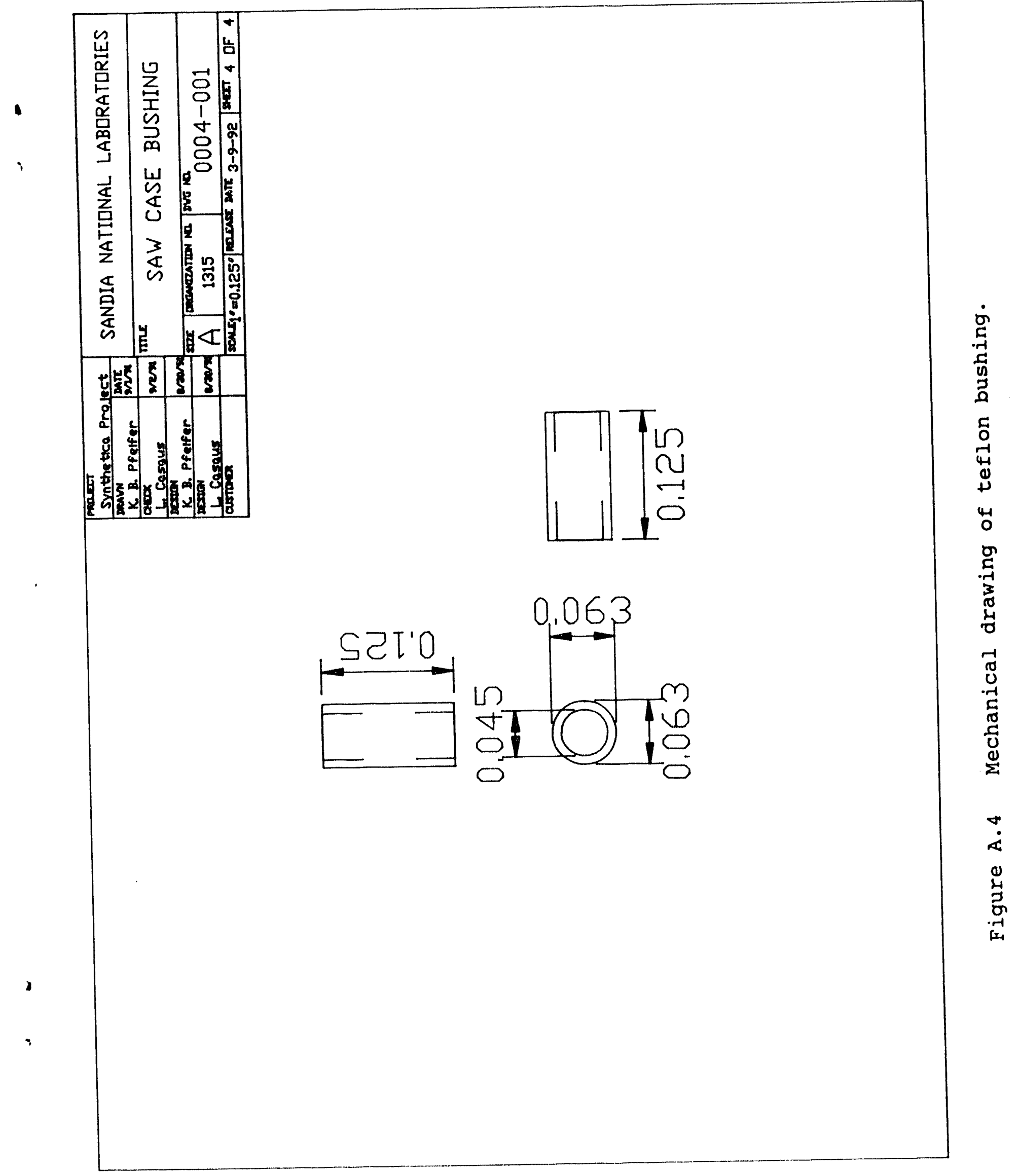




\section{REFERENCES}

1. T. R. Galloway, F. S. Howard, "On-site Reactivation of Granular Carbon with the Synthetica Detoxifier", 1991. Annual AIChE Meeting, Nov. 17-22, 1991.

2. H. Wohltjen, Sensors and Actuators, 1984, 5, 307.

3. G. C. Frye, S. J. Martin, R. W. Cernosek, and K. B. Pfeifer, "Portable Acoustic Wave Sensor systems for On-Line Monitoring of volatile Organics", International Journal of Environmentally Conscious Manufacturing, Vol. 1, No. 1, pp. 37-45, 1992.

4. R. M. White, Proc. IEEE, 58, 1238-1276 (1970).

5. ST-cut quartz is a singly rotated cut having Euler angles $\lambda=0^{\circ}, \theta=90^{\circ}$, and $\mu=132.75^{\circ}$.

6. S. J. Martin, A. J. Ricco, T. M. Niemczyk, and G. C. Frye, Sensors and Actuators, 20, 253, 1989.

7. S. Datta, Surface Acoustic Wave Devices, Prentice-Hall: Englewood Cliffs, NJ., 1986.

8. D. P. Morgan, Surface-Wave Devices for signal Processing", Elsevier, New York, New York, 1985.

9. V. M. Ristic, Principle of Acoustic Devices, Wiley, New York, New York, 1983, P. 127.

10. A. J. Slobodnik, E. D. Conway, and R. T. Delmonico, Microwave Acoustic Handbook, Vol. 1A. Surface Wave Velocities, National Technical Information Service, U. S. Dept. of Commerce, 1973.

11. Omega Engineering Inc., One Omega Drive, Box 4047, Stamford, CT 06907-0047.

12. Mini-circuits, P. O. Box 350166, Brooklyn, New York, $11235-0003$.

13. Avantek, 3175 Bowers Ave. Santa Clara, CA 95054.

14. Augat, 33 Perry Ave., P. O. Box 779, Attleboro, MA 02703.

15. Adams Russel Anzac, 80 Cambridge street, Burlington, MA, 01803 .

16. Analog Devices, one Technology Way, P.O. Box 9106, Norwood, MA, 02062-9106. 
17. Signetics Corporation, 811 E. Arques Ave., Sunnyvale, CA 94086 .

18. Newport Electronics Inc. 2229 South Yale Street, Santa Ana, $C A, 92704-4426$.

19. Dell Marketing Corp., 9505 Arboretum Blvd., Austin, TX 78759.

20. National semiconductor, 2900 Semiconductor Drive, P.O. Box 58090, Santa Clara, CA 95052-8090.

21. J. L. Graham, D. L. Hall, and B. Dellinger, "Laboratory Investigation of Thermal Degradation of a Mixture of Hazardous organic compounds. I", Environmental Science and Technology, Vol. 20, No. 7, Pg. 703-710, 1986. 
Distribution:

$\begin{array}{lll}1000 & \text { P. A. Fleury } \\ 1300 & \text { P. S. Peercy } \\ 1315 & \text { J. J. Wiczer } \\ 1315 & \text { S. J. Martin } \quad(15) \\ 1315 & \text { K. B. Pfeifer } \quad(15) \\ 1315 & \text { A. J. Ricco } \quad(15) \\ 1315 & \text { L. Casaus } \\ 1315 & \text { G. C. Frye } \\ 1315 & \text { R. W. Cernosek } \\ 6600 & \text { J. B. Woodard } \\ 6602 & \text { D. L. Berry } \\ 6602 & \text { J. L. Sprung } \\ 7141 & \text { Technical Library } \\ 7151 & \text { Technical Publications } \\ 7613-2 & \text { Document Processing DOE/OSTI } \\ 8523-2 & \text { Central Technical Files }\end{array}$



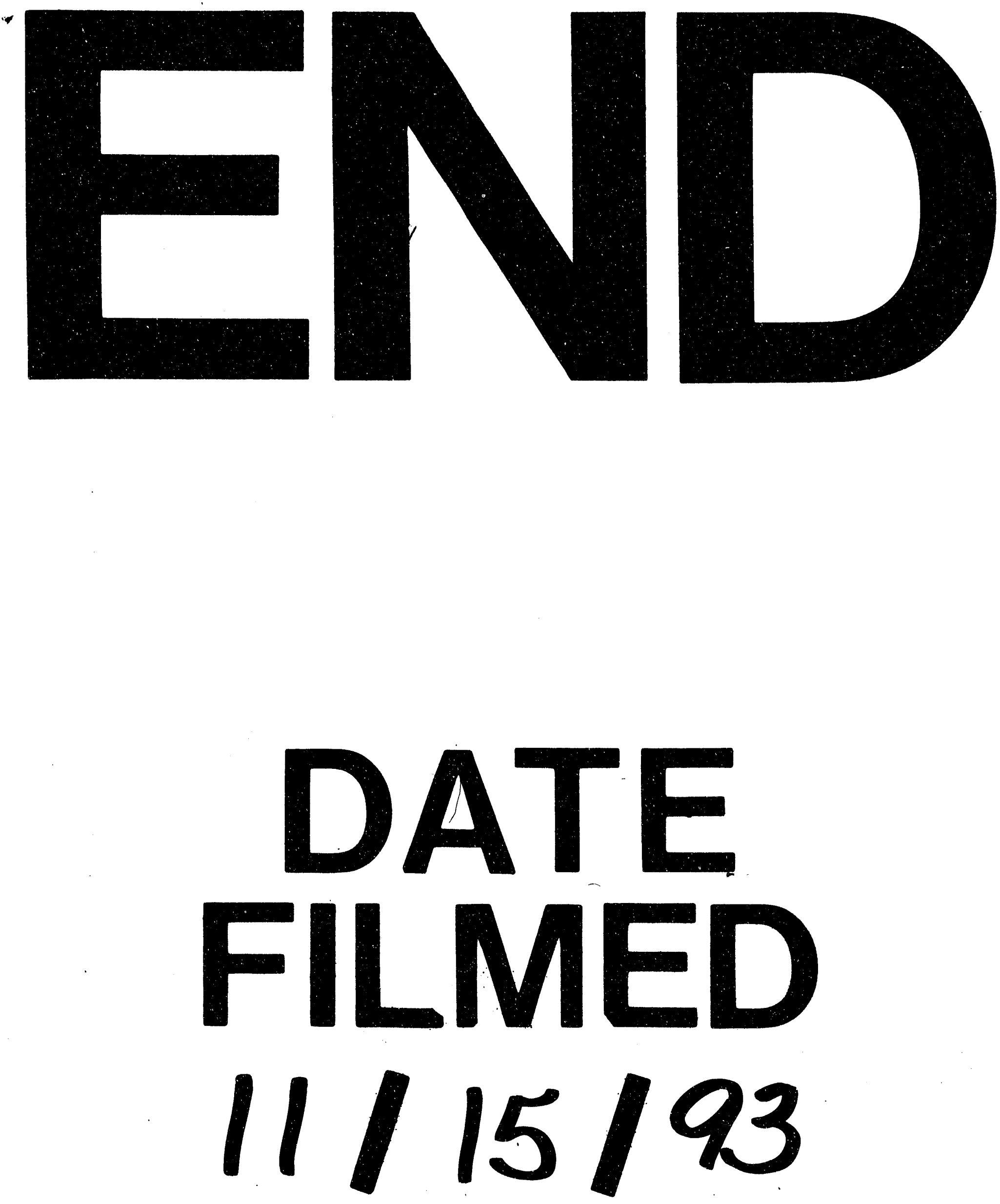
